\title{
Covariant description of kinetic freeze-out through a finite spacelike layer
}

\author{
E. Molnár, ${ }^{1}$ L. P. Csernai, ${ }^{1,2}$ V. K. Magas, ${ }^{1,3}$ A. Nyíri, ${ }^{1}$ and K. Tamosiunas ${ }^{1}$ \\ ${ }^{1}$ Section for Theoretical and Computational Physics and Bergen Computational Physics Laboratory, BCCS-Unifob, University of Bergen, \\ Allegaten 55, N-5007 Bergen, Norway \\ ${ }^{2}$ MTA-KFKI, Research Institute of Particle and Nuclear Physics, Post Office Box 49, H-1525 Budapest 114, Hungary \\ ${ }^{3}$ Departamento de Física Teórica and IFIC Centro Mixto, Universidad de Valencia-CSIC, Institutos de Investigación de Paterna, \\ Apdo. correos 22085, E-46071 Valencia, Spain
}

(Received 14 April 2005; revised manuscript received 13 February 2006; published 23 August 2006)

\begin{abstract}
The problem of freeze-out (FO) in relativistic heavy-ion reactions is addressed. We develop and analyze an idealized one-dimensional model of FO in a finite layer, based on the covariant FO probability. The resulting post FO phase-space distributions are discussed for different FO probabilities and layer thicknesses.
\end{abstract}

DOI: 10.1103/PhysRevC.74.024907

PACS number(s): 25.75.-q, 24.10.Nz

\section{INTRODUCTION}

The hydrodynamical description of relativistic particle collisions was first discussed more than 50 years ago by Landau [1] and nowadays it is frequently used in different versions for simulations of heavy-ion collisions. Such a simulation basically includes three main stages: the initial stage, the fluid-dynamical stage, and the so-called freeze-out (FO) stage when the hydrodynamical description breaks down. During this latter stage, the matter becomes dilute, cold, and noninteracting, the particles stream toward the detectors freely, and their momentum distribution freezes out. Thus, the FO stage is essentially the last part of a collision process and the source of the observables.

The usual recipe is to assume the validity of the hydrodynamical treatment up to a sharp FO hypersurface (e.g., when the temperature reaches a certain value, $T_{\mathrm{FO}}$ ). When we reach this hypersurface, all interactions cease and the distribution of particles can be calculated.

In such a treatment FO is a discontinuity where the properties of the matter change suddenly across some hypersurface in space-time. The general theory of discontinuities in relativistic flow was first discussed by Taub [2]. That description can only be applied to discontinuities across propagating hypersurfaces, which have a spacelike $\left(d \sigma_{\mu} d \sigma^{\mu}=-1\right)$ normal vector. The discontinuities across hypersurfaces with a timelike $\left(d \sigma_{\mu} d \sigma^{\mu}=1\right)$ normal vector were considered unphysical. The remedy for this came some 40 years later in [3], in which Taub's approach for both timelike and spacelike hypersurfaces was generalized. Consequently, it is possible to take into account conservation laws exactly across any surface of discontinuity in relativistic flow.

As was shown recently in Refs. [4,5], the frequently used Cooper-Frye prescription [6] to calculate post FO particle spectra gives correct results only for discontinuities across timelike normal vectors. The problem of negative contributions in the Cooper-Frye formula was healed by a simple cutoff, $\Theta\left(p^{\mu} d \sigma^{\mu}\right)$, proposed by Bugaev [4]. However, this formulation is still based on the existence of a sharp FO hypersurface, which is a strong idealization of a FO layer of finite thickness [7]. Thus, by assuming an immediate sharp
FO process, the questions of final-state interactions and the departure from local equilibrium are left unjustified.

The recent papers $[8,9]$ formulates the FO problem in the framework of kinetic transport theory. The dynamical FO description has to be based on the modified Boltzmann transport equation (MBTE), rather than on the commonly used Boltzmann transport equation (BTE). The MBTE abandons the local molecular chaos assumption and the requirement of smooth variation of the phase-space distribution, $f(x, p)$, in space-time. This modification of BTE makes it even more difficult to solve the FO problem from first principles. Therefore, it is very important to build phenomenological models, that can explain the basic features of the FO process.

The present paper aims at building such a simple phenomenological model. The kinetic approach presented is applicable for FO in a layer of finite thickness with a spacelike normal vector. It can be viewed as a continuation and generalization of Refs. [10-12]. The kinetic model for FO in the timelike direction was discussed in a recent paper [13]; however, the fully covariant model analysis and the treatment are presented in Ref. [14].

In the present work we use stationary, one-dimensional FO models for a transparent presentation. Such models can be solved semianalytically, allowing us to trace the effects of different model components, assumptions, and restrictions applied to the FO description. We do not aim at directly applying the results presented here to experimental heavy-ion collision data; instead our purpose is to study qualitatively the basic features of the FO process. We want to demonstrate the applicability of the proposed covariant FO escape rate and most importantly, to see the consequences of finishing FO in a finite layer. Up to now, two extreme ways of describing FO have been used; (i) FO on an infinitely narrow hypersurface and (ii) infinitely long FO in a volume emission type of model. To our knowledge this is the first attempt to, at least qualitatively, understand how FO in a finite space-time domain can be simulated and what will be its outcome. In such stationary, one-dimensional models the expansion cannot be realistically included, therefore it is ignored.

In realistic simulations of high-energy heavy-ion reactions the full three-dimensional (3D) description of a expanding and 
freezing out system should be included. This work is under initial development.

\section{FREEZE-OUT FROM KINETIC THEORY}

Kinetic theory describes the time evolution of a singleparticle distribution function $f(x, p)=f\left(t, \mathbf{x}, p^{0}, \mathbf{p}\right)$ in 6D phase-space. To describe FO in a kinetic model, we split the distribution function into two parts $[10,15,16]$ :

$$
f(x, p)=f_{i}(x, p)+f_{f}(x, p) .
$$

The free component, $f_{f}$, is the distribution of the frozenout particles, while $f_{i}$ is the distribution of the interacting particles. Initially, we have only the interacting part, then as a consequence of FO dynamics, $f_{i}$ gradually disappears, whereas $f_{f}$ gradually builds up. In this paper we convert the description of the FO process from a sudden FO (i.e., on a sharp hypersurface) into a gradual FO (i.e., in some finite space-time domain).

Freeze-out is known to be a strongly directed process [17], where the particles are allowed to cross the FO layer only outward, in the direction of the normal vector $d \sigma_{\mu}$ of the FO hypersurface. Many dynamical processes such as detonations, deflagrations, shocks, condensation waves, happen in a way where the phenomenon propagates in some direction. Basically, this means that the gradients of the described quantity (the distribution function in our case) in all perpendicular directions can be neglected compared to the gradient in the given direction $d \sigma_{\mu}$ (i.e., $\nabla f \approx d \sigma^{\mu} \partial_{\mu} f$ ). In such a situation these can be effectively described as one-dimensional processes, and the space-time domain, where such a process takes place, can be viewed as a layer.

Therefore, we develop a one-dimensional model for the FO process in a layer of finite thickness $L$. We assume that the boundaries of this layer are approximately parallel, and thus the thickness of the layer does not vary much. This can be justified, for example, in the case when the system size is much larger than $L$. At the inside boundary of this layer there are only interacting particles, whereas at the outside boundary all particles are frozen-out and no interacting particles remain. Note that the normal to the FO layer, $d \sigma^{\mu}$, can be spacelike or timelike.

The gradual FO model for the infinitely long onedimensional FO process was presented in recent works [10-12]. We are going to build a similar model, but now we make sure that FO is completely finished within a finite layer.

\section{A. Freeze-out in a finite layer}

In kinetic theory the interaction between particles is due to collisions. A quantitative characterization of collisions is given by the mean free path (mfp) $\lambda_{\text {mfp }}$, giving the average distance between collisons. The $\mathrm{mfp}$ is inversely proportional to the density: $\lambda_{\text {mfp }} \sim 1 / n(x)$. If we have a finite FO layer, the interacting particles inside this domain must have a finite mfp. During the FO process, as the density of the interacting particles decreases, they enter into a collisionless regime, where their final mfp tends to infinity or at least gets much larger than the system size $L$. The realistic FO process for nucleons in heavy-ion collisions happens within a finite space-time FO domain, which has a thickness of a few initial mfps [18]. Hence, one must realize that the FO process cannot be fully exploited by means of the mfp concept, since we have to describe a process where we have on average a few collisions per particle before FO. Therefore, this type of process should be analyzed by having another characteristic length scale different from the mfp. In our case it should be related to the thickness $L$ of the FO layer.

Based on strong flow and relatively small dissipation, it has recently been conjectured that the state where collective flow starts is strongly interacting and strongly correlated while the viscosity is not large [19]. This indicates a small $\mathrm{mfp}$, in the interacting matter, while at the surface $\lambda_{\text {mfp }} \rightarrow \infty$. Several indications point out that in high-energy heavy-ion reactions FO and hadronization happens simultaneously from a supercooled plasma [20-22]. This could be modeled in a way that prehadron formation and clusterization starts gradually in the plasma, and this process is coupled to FO in a finite layer. The FO is finished when the temperature of the interacting phase drops under a critical value and all quarks cluster into hadrons, which no longer collide. This is the possible qualitative scenario with well-defined finite thickness $L$ of the FO layer.

Now, let us recall the equations describing the evolution in the simple kinetic FO model [10-12]. Starting from a fully equilibrated Jüttner distribution $f_{J}(p)$ [i.e., $f_{i}(s=$ $0, p)=f_{J}(p)$ and $f_{f}(s=0, p)=0$, we have that the two components of the momentum distribution develop in the direction of the FO (i.e., along $d \sigma^{\mu}$ ) according to the following differential equations:

$$
\begin{gathered}
\partial_{s} f_{i}(s, p) d s=-f_{i}(s, p) W_{\mathrm{esc}}(s, p), \\
\partial_{s} f_{f}(s, p) d s=+f_{i}(s, p) W_{\mathrm{esc}}(s, p),
\end{gathered}
$$

where $W_{\mathrm{esc}}(s, p)$ is the escape rate governing the FO development and $s=x^{\mu} d \sigma_{\mu}$. Here $x^{\mu}$ is a four-vector having its origin $^{1}$ at the inner surface $S_{1}$ of the FO layer (see Fig. 1). To obtain the escape probability, for a particle passing from 0 to $s, \mathcal{P}_{\text {esc }}(s)$, we have to integrate the escape rate along a trajectory crossing the FO layer:

$$
f_{i}(s, p)=f_{J}(p) \underbrace{\exp \left(-\int_{0}^{s} d s^{\prime} W_{\mathrm{esc}}\left(s^{\prime}, p\right)\right)}_{1-\mathcal{P}_{\mathrm{esc}}} .
$$

The definition for the escape probability was previously given in Ref. [16], in terms of collision or scattering rates, where the FO process lasted an infinitely long time. In our finite-layer FO description the quantity that defines the escape probability is the escape rate.

To have a complete physical FO finished at a finite distance and time, we require $\mathcal{P}_{\text {esc }} \rightarrow 1$ when $s \rightarrow L$. In usual cascade

\footnotetext{
${ }^{1}$ Any point of the inner surface $S_{1}$ can be considered as an origin, since translations along $S_{1}$ do not change $s$, the projection of $x^{\mu}$ on the FO normal vector $d \sigma^{\mu}$, as long as $S_{1}$ and $S_{2}$ are parallel, as assumed. Of course, this latter assumption can be justified only locally, in some finite region, as is clear from Fig. 1.
} 


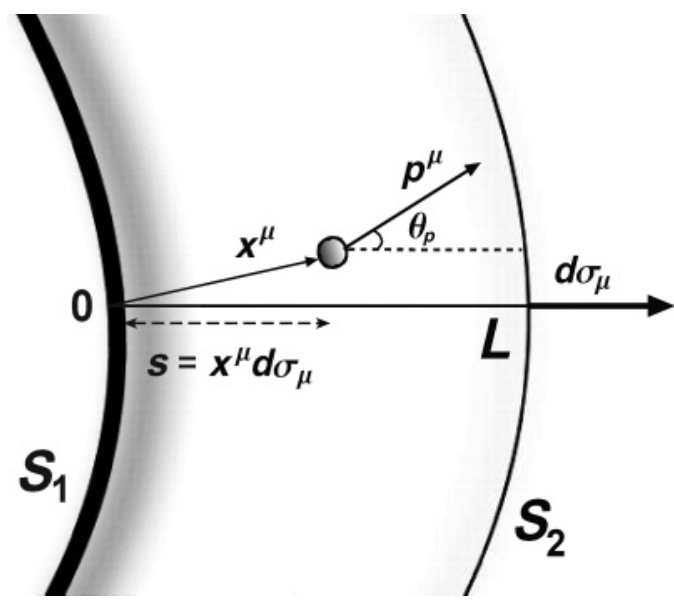

FIG. 1. The picture of a gradual FO process within the finite FO layer, in the $x$ direction [i.e., $\left.d \sigma_{\mu}=(0,1,0,0)\right]$. The particles are moving in different directions outward, indicated by the angle $\theta$. The inside boundary of the FO layer, $S_{1}$ (thick line), indicates the points where the FO starts. This is the origin of the coordinate vector $x^{\mu}$. Within the finite thickness of the FO layer, $L$, the density of the interacting particles gradually decreases (indicated by shading) and disappears at the outside boundary $S_{2}$ (thin line) of the FO layer.

models the probability of collision never becomes exactly zero, and correspondingly $\mathcal{P}_{\text {esc }}$ never becomes exactly one, and the FO process lasts ad infinitum. This is because the probability of collision is calculated based on the thermally averaged cross section, which does not vanish for thermal (e.g., Gaussian) momentum distributions. In reality the free or frozen-out particles have no isotropic thermal distributions but these distributions can be anisotropic and strongly confined in phase-space. This means that the collision probability can be exactly zero and FO may be completed in a finite space-time domain.

It seems reasonable to parametrize the escape rate, which has dimension one over length, in terms of some characteristic FO length $\lambda^{\prime}(s, p)$ :

$$
W_{\mathrm{esc}}\left(x^{\mu}, p^{\mu}, d \sigma_{\mu}\right) \equiv \frac{1}{\lambda^{\prime}(s, p)} \Theta\left(p^{s}\right),
$$

where the cutoff factor $\Theta\left(p^{s}\right) \equiv \Theta\left(p^{\mu} d \sigma_{\mu}\right)$ forbids the FO of particles with momenta not pointing outward [4]. This FO parameter, $\lambda^{\prime}(s, p)$, is not necessarily an average distance in space or duration in time between two subsequent collisions, like the $\mathrm{mfp}$. The $\mathrm{mfp}$ tends to infinity as the density decreases, while the FO just becomes faster in this limit. Actually, the FO scale behaves in the opposite way to the $\mathrm{mfp}$. This can be seen, for example, in a simple, purely geometrical FO model, which takes into account the divergence of the flow in a $3 \mathrm{D}$ expansion [23]. Both this and the phase transition or clusterization effect described at the beginning of this section lead to a finite FO layer $L$, even if the $\operatorname{mfp}\left(\lambda_{\operatorname{mfp}} \cong 1 / n \sigma\right)$ is still finite at the outer edge of this layer.

We consider the thickness of the layer $L$ to be the "proper" thickness of the FO layer, because it depends only on invariant scalar matter properties such as cross section, proper density, velocity divergence, phase transition, or clusterization rates.
These should be evaluated in the local rest (LR) frame of the matter, and, since the layer is finite, around the middle of this layer. The proper thickness is analogous to the proper time (i.e., time measured in the rest frame of the particle); hence the proper thickness is the thickness of the FO layer measured in the rest frame attached to the FO front, that is, the rest frame of the front (RFF). Some of the parameters, such as the velocity divergence and the phase transition rate, describe the dynamical changes in the layer, so these can determine the properties (e.g., the thickness) of the finite layer. However, calculating $L$ from these mentioned properties is beyond the scope of this paper, and $L$ is treated as a parameter in the following.

Let us consider the RFF, where the normal vector of the front points either in the time $(t)$ or in the space $(x)$ direction, introducing the following notations. ${ }^{2}$ Indeed, if $d \sigma_{\mu}$ is spacelike the resulting equations can be transformed into a frame where the process is stationary [here $d \sigma_{\mu}=(0,1,0,0)$ and correspondingly $s \equiv x$ )], while in the case of a timelike normal vector the equations can be transformed into a frame where the process is uniform and time dependent $\left[d \sigma_{\mu}=\right.$ $(1,0,0,0), s \equiv t]$. For the sake of transparency and simplicity we will perform calculations only for FO in a finite layer with a spacelike normal vector in this paper, but many intermediate results can and will be obtained in a Lorentz-invariant way.

Inside the FO layer particles are separated into stillcolliding or interacting and not-colliding or free particles. The probability not to collide with anything on the way out should depend on the number of particles, in the way of a particle moving outward in the direction $\mathbf{p} /|\mathbf{p}|$ across the FO layer of thickness $L$ (see Fig. 1). If we follow a particle moving outward form the beginning $\left(x^{\mu}=0\right)$, that is, the inner surface of the FO layer, $S_{1}$, to a position $x^{\mu}$, there is still a distance

$$
\frac{L-s}{\cos \theta_{p}}
$$

ahead of us, where $\theta_{\mathbf{p}}$ is the angle between the normal vector and $\mathbf{p} /|\mathbf{p}|$. As this remaining distance becomes smaller the probability to freeze-out becomes larger; thus, we may assume that the escape rate is inversely proportional to some power $a$ of this quantity [9,24].

Based on these assumptions we write the escape rate as

$$
W_{\mathrm{esc}}=\frac{1}{\lambda}\left(\frac{L}{L-s}\right)^{a}\left(\cos \theta_{\mathbf{p}}\right)^{a} \Theta\left(p^{s}\right),
$$

where this newly introduced parameter $\lambda$ is the initial (i.e., at $S_{1}$ ) characteristic FO length of the interacting matter, $\lambda=\lambda^{\prime}\left(s=0, \cos \theta_{\mathbf{p}}=1\right)$. The power $a$ influences the FO profile across the front. Indeed, calculating the escape probability

$\begin{array}{lcc} & & \\ & & \\ & \text { Timelike } & \text { Spacelike } \\ s \equiv\left(d \sigma_{\mu} x^{\rho}\right) & (1,0,0,0) & (0,1,0,0) \\ p^{s} \equiv\left(d \sigma_{\rho} p^{\rho}\right) & p^{0} & x \\ \partial_{s} \equiv\left(d \sigma^{\rho} \partial_{\rho}\right) & \partial_{t} & p^{x} \\ \lambda^{\prime}(s) & \tau^{\prime}(t) & \partial_{x} \\ & & \lambda^{\prime}(x)\end{array}$


$\mathcal{P}_{\text {esc }}$, Eq. (3), with the escape rate, given by Eq. (5), we find

$$
\mathcal{P}_{\text {esc }}=1-\left(\frac{L-s}{L}\right)^{\frac{L}{\lambda} \cos \theta_{\mathbf{p}} \Theta\left(p^{s}\right)},
$$

for $a=1$, and

$$
\mathcal{P}_{\text {esc }}=1-\exp \left[\frac{L}{\lambda} \Theta\left(p^{s}\right) \frac{\left(\cos \theta_{\mathbf{p}}\right)^{a}}{(a-1)}\left(1-\left(\frac{L}{L-s}\right)^{a-1}\right)\right]
$$

for $a \neq 1$. Thus, we see that for different $a$ values we have different FO profiles:

$a=1$ : powerlike $\mathrm{FO}$,

$a>1$ : fast, exponential-like FO,

$a<1$ : no complete FO within the finite layer, since $\mathcal{P}_{\text {esc }}$ does not tend to 1 as $s$ approaches $L$.

In papers [10-12] the authors were using $a=1$ and were modeling FO in an infinite layer. To study the effects of FO within a finite space-time domain, we would like to compare the results of our calculations with those of earlier works; therefore we shall also take $a=1$ in further calculations. It is easy to check that our escape rate, Eq. (5), equals the earlier expression

$$
P_{\mathrm{esc}}=\frac{\cos \theta_{\mathbf{p}}}{\lambda} \Theta\left(p^{s}\right)
$$

in the $L \rightarrow \infty$ limit. Thus, the model discussed in this paper is a generalization of the models for infinitely long FO, described in [10-12], and allows us to study FO in a layer of finite thickness.

The angular factor $\cos \theta_{\mathbf{p}}$ maximized the FO probability for those particles, that propagate in the direction closest to the normal of the FO layer. For the FO in timelike directions, studied in Ref. [13], the angular factor was 1. This factor, and correspondingly the escape rate, Eq. (5), are not covariant. Furthermore, this earlier formulation does not take into account that the escape rate of particles should be proportional to the particle velocity (since the conventional nonrelativistic limit of the collision rate contains the thermal average $\langle\sigma v\rangle)$. Let us consider the simplest situation, when the RFF is the same as the rest frame of the gas (RFG), where the flow velocity is $u^{\mu}=(1,0,0,0)$. If FO propagates in a spacelike direction [i.e., $d \sigma_{\mu}=(0,1,0,0)$ ], as shown in Fig. 1, then $\cos \theta_{\mathbf{p}}=p^{x} /|\mathbf{p}|$. Therefore, a straightforward generalization of the escape rate, based on these arguments, is

$$
\cos \theta_{\mathbf{p}} \times|\mathbf{v}| \equiv \frac{p^{x}}{|\mathbf{p}|} \times \frac{|\mathbf{p}|}{p^{0}}=\left(\frac{p^{\mu} d \sigma_{\mu}}{p^{\mu} u_{\mu}}\right)
$$

where the right-hand side of this equation is an invariant scalar in covariant form. Now, we assume that this simple generalization is valid for any spacelike or timelike FO direction, even when the RFG and RFF are different $[9,17,25]$.
Based on these arguments, we can write the total escape rate from Eq. (5) in a Lorentz-invariant ${ }^{3}$ form:

$$
W_{\mathrm{esc}}=\frac{1}{\lambda}\left(\frac{L}{L-s}\right)\left(\frac{p^{s}}{p^{\mu} u_{\mu}}\right) \Theta\left(p^{s}\right),
$$

which now opens room for a general study of FO in relativistic flow in layers of any thickness.

Former FO calculations in Refs. [10-12] were always performed in the RFF. Aiming for semianalytical results and transparent presentation, as well as to compare our results with former calculations, we will also study the system evolution in the RFF, but now this is only our preference. In principle calculations can be performed in any reference frame. In more realistic many-dimensional models, which will take into account the system expansion simultaneous with the gradual FO, it will be probably more adequate to work in the RFG or in the lab frame, and our invariant escape rate, Eq. (7), can be directly used as a basic FO ingredient of such models.

\section{B. The Lorentz-invariant escape rate}

In this section let us study this new angular factor in more detail. We will take the $p$-dependent part of the escape rate, Eq. (7), and denote it as

$$
W(p)=\frac{p^{\mu} d \sigma_{\mu}}{p^{\mu} u_{\mu}} \Theta\left(p^{\mu} d \sigma_{\mu}\right) .
$$

In the RFG, where the flow velocity of the matter is $u^{\mu}=$ $(1,0,0,0)_{\mathrm{RFG}}$ by definition, $W(p)$ is given as

$$
W(p)=\left.\frac{p^{\mu} d \sigma_{\mu}}{p^{0}} \Theta\left(p^{\mu} d \sigma_{\mu}\right)\right|_{\mathrm{RFG}},
$$

and it is smoothly changing as the direction of the normal vector changes in the RFG. This will be discussed in more detail in the rest of this section.

Now, we will take different typical points of the FO hypersurface, A, B, C, D, E, and F (see Fig. 2). At these points, the normal vectors of the hypersurface, $d \sigma_{\mu}=(h, i, j, k)_{\mathrm{RFG}}$, are given in the following.

To calculate the normal vector for the different cases shown in Fig. 2, we simply make use of the Lorentz transformation. The normal vector of the timelike part of the FO hypersurface may be defined as the local $t^{\prime}$ axis, while the normal vector of the spacelike part may be defined as the local $x^{\prime}$ axis. As $d \sigma_{\mu}$ is normalized to unity, its components may be interpreted in terms of $\gamma_{\sigma}$ and $v_{\sigma}$, where $\gamma_{\sigma}=1 / \sqrt{1-v_{\sigma}^{2}}$. So, we have

(A) $d \sigma_{\mu}=(1,0,0,0)$, which leads to $W(p)=1$;

(B) $d \sigma_{\mu}=\gamma_{\sigma}\left(1, v_{\sigma}, 0,0\right)$, which leads to $W(p)=$ $\gamma_{\sigma}\left(p^{0}+v_{\sigma} p^{x}\right) / p^{0}$

(C) $d \sigma_{\mu}=\gamma_{\epsilon}(1+\epsilon, 1-\epsilon, 0,0)$, where $\gamma_{\epsilon}=(4 \epsilon)^{-\frac{1}{2}}$, $\epsilon \ll 1$; this leads to $W(p)=\gamma_{\epsilon}\left[\left(p^{0}+p^{x}\right)+\right.$ $\left.\epsilon\left(p^{0}-p^{x}\right)\right] / p^{0}$

\footnotetext{
${ }^{3}$ Now, it is important that $L$ is defined as an invariant scalar, so $W_{\text {esc }}$ is also an invariant scalar.
} 


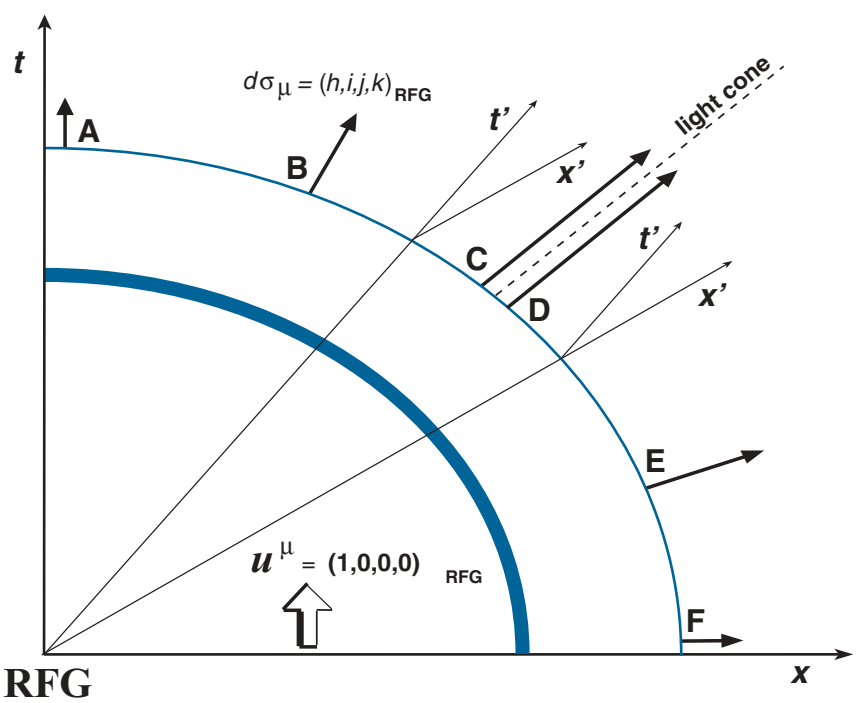

FIG. 2. (Color online) A simple FO hypersurface $[t, x]$ in the RFG, where $u^{\mu}=(1,0,0,0)_{\mathrm{RFG}}$, including timelike and spacelike parts. The normal vector of the FO front, $d \sigma_{\mu}$, is a timelike four-vector at the timelike part and it is changing smoothly into a spacelike fourvector in the spacelike part. On these two parts of the hypersurface, in the RFF, $d \sigma_{\mu}$ points in the direction of the $t^{\prime}$ and $x^{\prime}$ axes, respectively. At points A, B, C, D, E, and F, we have different REFs ([t $\left.\left.t^{\prime}, x^{\prime}\right]\right)$.

(D) $d \sigma_{\mu}=\gamma_{\epsilon}(1-\epsilon, 1+\epsilon, 0,0)$, where $\gamma_{\epsilon}=(4 \epsilon)^{-\frac{1}{2}}, \epsilon \ll$ 1 ; this leads to $W(p)=\left\{\gamma_{\epsilon}\left[\left(p^{0}+p^{x}\right)-\epsilon\left(p^{0}-\right.\right.\right.$ $\left.\left.\left.p^{x}\right)\right] / p^{0}\right\} \Theta\left[\gamma_{\epsilon}\left(p^{0}+p^{x}\right)-\gamma_{\epsilon} \epsilon\left(p^{0}-p^{x}\right)\right]$

(E) $d \sigma_{\mu}=\gamma_{\sigma}\left(v_{\sigma}, 1,0,0\right)$, which leads to $W(p)=$ $\left[\gamma_{\sigma}\left(v_{\sigma} p^{0}-p^{x}\right) / p^{0}\right] \Theta\left[\gamma_{\sigma}\left(v_{\sigma} p^{0}-p^{x}\right)\right] ;$ and

(F) $d \sigma_{\mu}=(0,1,0,0)$, which leads to $W(p)=$ $\left(p^{x} / p^{0}\right) \Theta\left(p^{x}\right)$.
The resulting phase-space escape rates are shown in Fig. 3 for these six cases.

Similar calculations can be done in the RFF, where $d \sigma_{\mu}=$ $(1,0,0,0)$ for $\mathrm{A}, \mathrm{B}$, and $\mathrm{C}$ and $d \sigma_{\mu}=(0,1,0,0)$ for $\mathrm{D}, \mathrm{E}$, and $\mathrm{F}$, leading to the following:

(A) $u_{\mu}=(1,0,0,0)$, which leads to $W(p)=1$;

(B) $u_{\mu}=\gamma_{\sigma}\left(1,-v_{\sigma}, 0,0\right)$, which leads to $W(p)=$ $p^{0} /\left[\gamma_{\sigma}\left(p^{0}-v_{\sigma} p^{x}\right)\right]$

(C) $u_{\mu}=\gamma_{\epsilon}[1+\epsilon,-(1-\epsilon), 0,0]$, where $\gamma_{\epsilon}=(4 \epsilon)^{-\frac{1}{2}}$, $\epsilon \ll 1$; this leads to $W(p)=\left(1 / \gamma_{\epsilon}\right) p^{0} /\left[\left(p^{0}-p^{x}\right)+\right.$ $\left.\epsilon\left(p^{0}+p^{x}\right)\right]$

(D) $u_{\mu}=\gamma_{\epsilon}[1+\epsilon,-(1-\epsilon), 0,0]$, where $\gamma_{\epsilon}=(4 \epsilon)^{-\frac{1}{2}}$, $\epsilon \ll 1$; this leads to $W(p)=\left(1 / \gamma_{\epsilon}\right) p^{0} /\left[\left(p^{0}-p^{x}\right)+\right.$ $\left.\epsilon\left(p^{0}+p^{x}\right)\right] \times \Theta\left(p^{x}\right)$

(E) $u_{\mu}=\gamma_{\sigma}\left(1,-v_{\sigma}, 0,0\right)$, which leads to $W(p)=$ $p^{x} /\left[\gamma_{\sigma}\left(p^{0}-v_{\sigma} p^{x}\right)\right] \times \Theta\left(p^{x}\right) ;$ and

(F) $u_{\mu}=(1,0,0,0)$, which leads to $W(p)=p^{x} / p^{0} \Theta\left(p^{x}\right)$.

For cases, A, B, C, D, E, and F in the RFF the resulting phase-space escape rates are shown in Fig. 4.

Figures 3 and 4 show that the momentum dependence of the escape rate, uniform in point $\mathrm{A}$, becomes different at different points of the FO hypersurface, but this change is continuous, when we cross the light cone, from point $\mathrm{C}$ to point D. Although in the RFF (Fig. 4) it seems that there is a principal difference between spacelike and timelike FO directions, owing to the cutoff $\Theta\left(p^{\mu} d \sigma_{\mu}\right)$ function, this is only a consequence of the chosen reference frame; that is, the RFF is defined in a way to stress the difference between these two cases, since in going from $\mathrm{C}$ to $\mathrm{D}$, the normal vector has a jump [i.e., $d \sigma_{\mu}=(1,0,0,0)$ goes over to $\left.d \sigma_{\mu}=(0,1,0,0)\right]$. Nevertheless, $W(p)$ is a continuous function as we change $d \sigma_{\mu}$, and in other frames, for example in the RFG (Fig. 3), we can see this clearly.
A

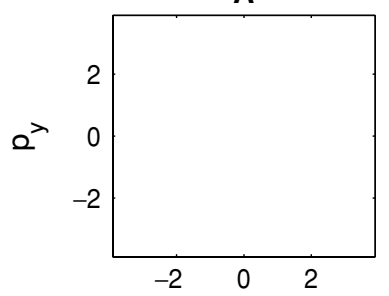

D

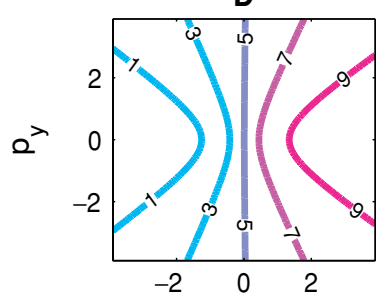

B

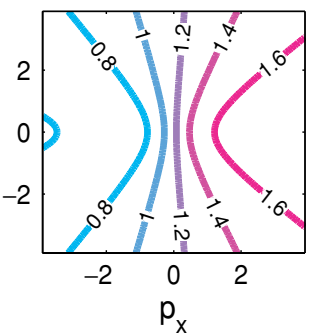

$\mathbf{E}$

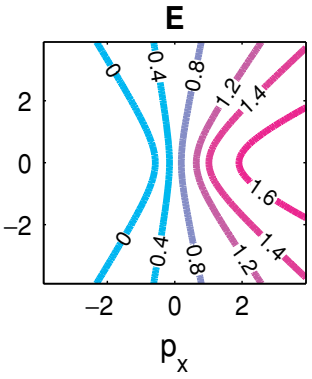

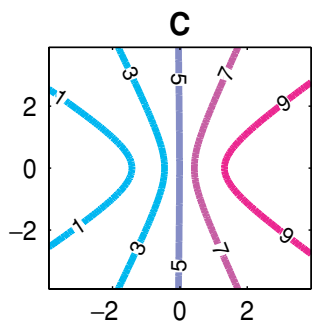

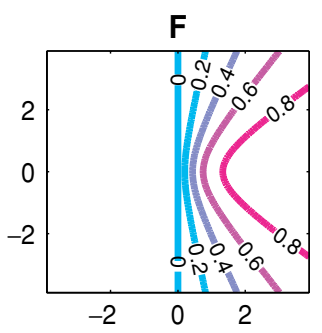

FIG. 3. (Color online) The contour plots of the momentum-dependent part of the escape rate, $W(p)$, as in Eq. (9), presented in six subplots at different points of the FO hypersurface. All plots are in the RFG. For region $\mathrm{A}, d \sigma_{\mu}=(1,0,0,0)$, and $W(p)$ is one uniformly; for $\mathrm{B}, d \sigma_{\mu}=\gamma_{\sigma}(1,0.5$, $0,0)$, for $\mathrm{C}, d \sigma_{\mu}=\gamma_{\epsilon}(1.01,0.99,0,0)$; for D, $d \sigma_{\mu}=\gamma_{\epsilon}(0.99,1.01,0,0) ;$ for $\mathrm{E}, \quad d \sigma_{\mu}=$ $\gamma_{\sigma}(0.5,1,0,0)$; for $\mathrm{F}, d \sigma_{\mu}=(0,1,0,0)$. The momenta are in units of particle mass, $[\mathrm{m}]$. 
A
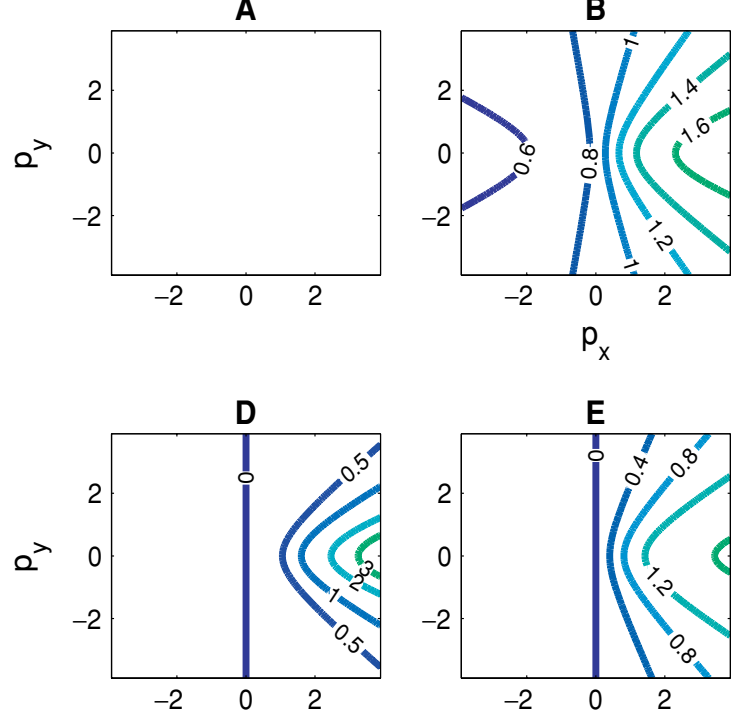

B

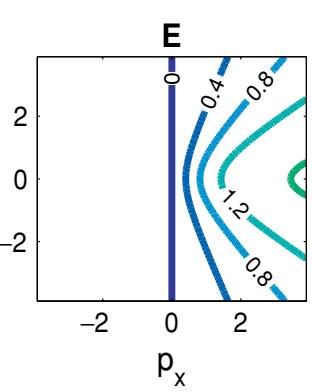

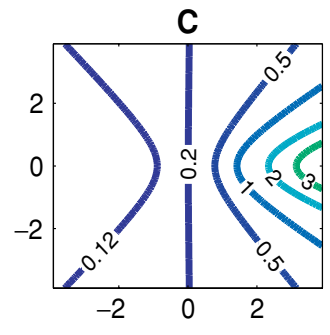

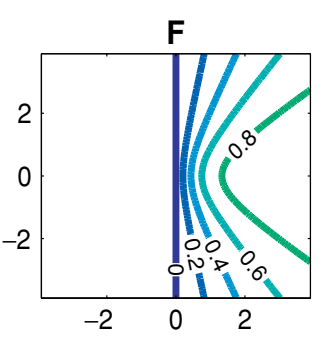

FIG. 4. (Color online) The contour plots of the momentum-dependent part of the escape rate, $W(p)$, in the RFF. For region $\mathrm{A}, \quad u_{\mu}=(1,0,0,0), \quad$ and $W(p)$ is one uniformly; for $\mathrm{B}, u_{\mu}=\gamma_{\sigma}(1,-0.5,0,0)$; for $\mathrm{C}, u_{\mu}=\gamma_{\epsilon}(1.01,-0.99,0,0)$; for $\mathrm{D}, u_{\mu}=$ $\gamma_{\epsilon}(1.01,-0.99,0,0)$; for $\mathrm{E}, u_{\mu}=\gamma_{\sigma}(1,-0.5$, $0,0)$; for $\mathrm{F}, u_{\mu}=(1,0,0,0)$. In cases $\mathrm{D}, \mathrm{E}$, and $\mathrm{F}$ the escape rate vanishes for momenta with $p_{x}<0$. The momenta are in units of particle mass, $[m]$.

\section{The updated simple kinetic model}

Now, using the new invariant escape rate, Eq. (7), we can generalize the simple model presented in [10-12] [i.e., Eqs. (2)] for a finite space-time FO layer:

$$
\begin{aligned}
& \partial_{s} f_{i} d s=-\left(\frac{L}{L-s}\right)\left(\frac{p^{s}}{p^{\mu} u_{\mu}}\right) \Theta\left(p^{s}\right) f_{i} \frac{d s}{\lambda}, \\
& \partial_{s} f_{f} d s=\left(\frac{L}{L-s}\right)\left(\frac{p^{s}}{p^{\mu} u_{\mu}}\right) \Theta\left(p^{s}\right) f_{i} \frac{d s}{\lambda} .
\end{aligned}
$$

Solving the first equation we find for the interacting component

$$
\begin{aligned}
f_{i}(s, p) & =f_{J}(p)\left(\frac{L-s}{L}\right)^{\frac{L}{\lambda}\left(\frac{p^{s}}{p^{p} u_{\mu}}\right) \Theta\left(p^{s}\right)} \\
& \stackrel{s \rightarrow L}{\longrightarrow} f_{J}(p) \Theta\left(-p^{s}\right) .
\end{aligned}
$$

Now, inserting this result into the second differential equation, from Eqs. (10), we obtain the FO solution, which describes the momentum distribution of the frozen-out particles:

$$
\begin{aligned}
f_{f}(s, p) & =f_{J}(p)\left[1-\left(\frac{L-s}{L}\right)^{\frac{L}{\lambda}\left(\frac{p^{s}}{p^{\mu_{u}} u_{\mu}}\right) \Theta\left(p^{s}\right)}\right] \\
& \stackrel{s \rightarrow L}{\longrightarrow} f_{J}(p) \Theta\left(p^{s}\right) .
\end{aligned}
$$

As $s$ tends to $L$ (i.e., to the outer boundary of the FO layer), this distribution, depending on the direction of the normal vector (spacelike or timelike) will tend to the cut-Jüttner or Jüttner distribution. The cut-Jüttner distribution means that part of the original Jüttner distribution survives even when we reach the outer boundary of the FO surface. To remedy this highly unrealistic result, in Refs. [10-13], rethermalization in the interacting component was taken into account via the relaxation time approximation; that is, we insert into the equation for the interacting component a new term, that describes how the interacting component approaches some equilibrated (Jüttner) distribution $f_{\text {eq }}(s)$ with a relaxation length $\lambda_{0}$ :

$$
\begin{aligned}
\partial_{s} f_{i} d s= & -\left(\frac{L}{L-s}\right)\left(\frac{p^{s}}{p^{\mu} u_{\mu}}\right) \Theta\left(p^{s}\right) f_{i} \frac{d s}{\lambda} \\
& +\left[f_{\text {eq }}(s)-f_{i}\right] \frac{d s}{\lambda_{0}}, \\
\partial_{s} f_{f} d s= & \left(\frac{L}{L-s}\right)\left(\frac{p^{s}}{p^{\mu} u_{\mu}}\right) \Theta\left(p^{s}\right) f_{i} \frac{d s}{\lambda} .
\end{aligned}
$$

Let us concentrate on the equation for the interacting component. Here the first term from Eq. (13), related to FO, moves the distribution out of equilibrium and decreases the energy-momentum density and baryon density of the interacting particles. The second term from Eq. (13) changes the distribution in the direction of the thermalization, although it does not affect the conserved quantities. The relative strength of the FO and rethermalization processes is determined by the two characteristic lengths $\lambda$ and $\lambda_{0}$.

In general the evolution of the interacting component can be solved numerically or semianalytically, at every step of the integration. Then, the change of conserved quantities caused by FO should be evaluated using the actual distribution $f_{i}(s, p)$ at the corresponding point $s$. For the purpose of this work, namely for the qualitative study of the FO features, it is enough to use an approximate solution, similarly as was done in [1113]. This would also allow us to make a direct comparison with results of these older calculations. Thus, the evaluation of the change of the conserved quantities is done analytically; that is, $f_{i}(s, p)$ is approximated with an equilibrium distribution function $f_{\mathrm{eq}}(s)$ with parameters $T(s), n(s)$, and $u^{\mu}(s)$.

This approximation is based on the fact that in most physical situations the overall number of particle collisions vastly exceeds the number of those collisions after which a particle leaves the system or freezes out. This allows us to assume that rethermalization ${ }^{4}$ happens faster than the freeze

\footnotetext{
${ }^{4}$ The words "immediate rethermalization" used in a few earlier publications were badly chosen, misleading, and inappropriate.
} 


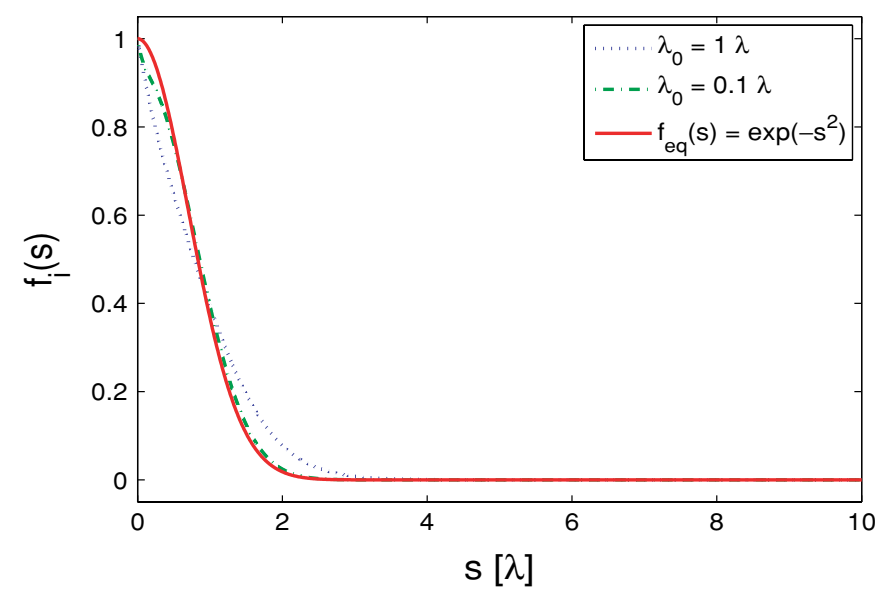

FIG. 5. (Color online) Numerical solutions of Eq. (13) for different $\lambda_{0}$ 's. This solution was obtained a using $f_{\text {eq }}=e^{-s^{2}}$ test function, for $L=10 \lambda$. The results show that $f_{i}(s)$ approaches $f_{\text {eq }}(s)$ when $\lambda_{0} \ll \lambda$.

out (i.e., that $\lambda_{0}<\lambda$ or $\lambda_{0} \ll \lambda$ ). Of course, this argument is true only at the beginning of the FO process, when the density of the interacting particles is still large. When $s$ is close to $L$ (i.e., near the outer hypersurface), the first term in Eq. (13) becomes more important than the rethermalization term because of its denominator, but, as we shall see in the results section, particles freeze-out exponentially fast and for large $s$, when say $99 \%$ of the matter is frozen out, the error we introduce with our approximate solution can not really affect the physical situation.

For illustration let us take a test function, $f_{\mathrm{eq}}(s)=e^{-s^{2}}$ (ignoring the $p$ dependence for the moment), that is a smoothly and fastly decreasing function. ${ }^{5}$ In Fig. 5 we show the numerical solutions for the interacting component for $\lambda_{0}=\lambda$ and $\lambda_{0}=0.1 \lambda$. The results show that for the latter case we can safely take the approximate solution [11-13]:

$$
f_{i}(s)=f_{\mathrm{eq}}(s)
$$

\section{Conservation laws}

The goal of the FO calculations is to find the final post FO momentum distribution, and then the corresponding quantities defined through it, starting from the initial pre FO distribution. On the pre FO side we can have equilibrated matter or gas. Its local rest frame defines the RFG (see Fig. 6). We can also define the reference frame, which is attached to the FO front, namely the RFF (see Fig. 7). These choices are usually advantageous, but other choices are also possible.

\footnotetext{
${ }^{5}$ In real calculations the $s$ dependence of $f_{\text {eq }}(s, p)$ is calculated from the energy, momentum, and baryon charge loss of the interacting component, where these losses are determined by the momentum dependence of the escape rate and the actual shape of $f_{i}(s, p)$, as discussed here.
}

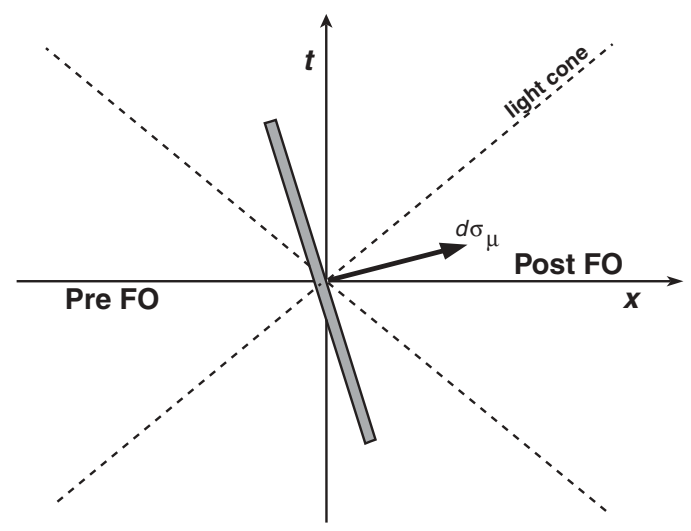

FIG. 6. The orientation of the freeze-out front in the RFG is given as $d \sigma_{\mu}=\gamma_{\sigma}\left(v_{\sigma}, 1,0,0\right)_{\mathrm{RFG}}$.

Furthermore, the conservation laws and the nondecreasing entropy condition must be satisfied [10]:

$$
\left[N^{\mu} d \sigma_{\mu}\right]=0, \quad\left[T^{\mu v} d \sigma_{\mu}\right]=0, \quad\left[S^{\mu} d \sigma_{\mu}\right] \geqslant 0,
$$

where $[A]=A-A_{0}$. The pre FO side baryon and entropy currents and the energy-momentum tensor are denoted by $N_{0}^{\mu}, S_{0}^{\mu}, T_{0}^{\mu \nu}$, while the post FO quantities are denoted by $N^{\mu}, S^{\mu}, T^{\mu \nu}$.

The change of conserved quantities caused by the particle transfer from the interacting matter to the free matter can be obtained in the following way. For the conserved particle four-current we have

$$
d N^{\mu}=d N_{i}^{\mu}+d N_{f}^{\mu}=0 \Rightarrow d N_{i}^{\mu}=-d N_{f}^{\mu} .
$$

Then, using the kinetic definition of the particle current, together with Eqs. (14) and (15), we obtain

$$
\begin{aligned}
d N_{i}^{\mu}(s) & =d s \int \frac{d^{3} p}{p^{0}} p^{\mu}\left[\partial_{s} f_{i}\right] \\
& =-\frac{d s}{\lambda}\left(\frac{L}{L-s}\right) \int \frac{d^{3} p}{p^{0}} p^{\mu}\left[\frac{p^{s}}{p_{\rho} u_{i}^{\rho}} \Theta\left(p^{s}\right)\right] f_{\mathrm{eq}}(s) .
\end{aligned}
$$

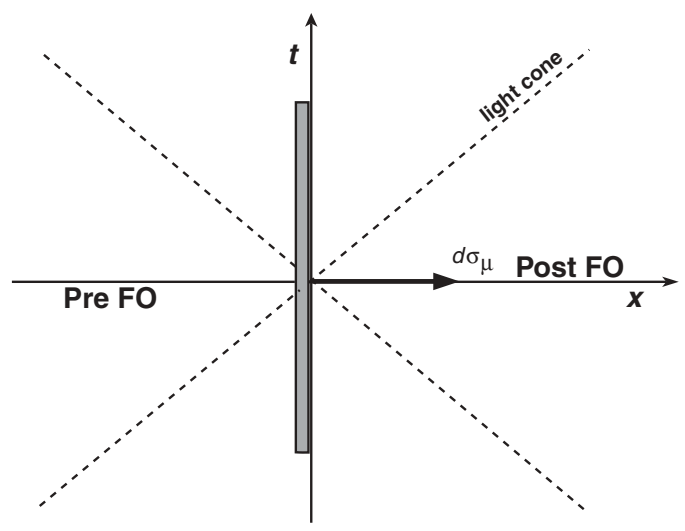

FIG. 7. The orientation of the freeze-out front in the RFF (i.e. in its own rest frame) is $d \sigma_{\mu}=(0,1,0,0)_{\mathrm{RFF}}$. In this frame the gas has nonvanishing velocity in general. 
Similarly, the change in the energy-momentum is

$$
d T_{i}^{\mu v}(s)=-\frac{d s}{\lambda}\left(\frac{L}{L-s}\right) \int \frac{d^{3} p}{p^{0}} p^{\mu} p^{v}\left[\frac{p^{s}}{p_{\rho} u_{i}^{\rho}} \Theta\left(p^{s}\right)\right] f_{\mathrm{eq}}(s) .
$$

The parameters of the equilibrium (Jüttner) distribution $f_{\text {eq }}(s)$ have to be recalculated after each step $d s$ from the conservation laws as in [11-13].

The change of flow velocity, $d u_{i}^{\mu}(s)$, can be calculated using Eckart's or Landau's definition of the flow, that is, from $d N_{i}^{\mu}(s)$ or $d T_{i}^{\mu \nu}(s)$, correspondingly. Then the change of conserved particle density is given by

$$
d n_{i}(s)=u_{i}^{\mu}(s) d N_{i}^{\mu}(s),
$$

and for the change of energy density we have

$$
d e_{i}(s)=u_{\mu, i}(s) d T_{i}^{\mu \nu}(s) u_{v, i}(s) .
$$

The change of the temperature of interacting component can be found from this last equation, Eq. (21), and from the equation of state (EoS). This closes our system of equations.

If we fix the FO direction to the $x$ direction, then Eqs. (18) and (19) can be rewritten as

$$
\begin{aligned}
d N_{i}^{\mu}(x)= & -\frac{d x}{\lambda}\left(\frac{L}{L-x}\right) \int \frac{d^{3} p}{p^{0}} p^{\mu} \\
& \times\left[\frac{p \cos \theta_{\mathbf{p}}}{\gamma\left(p^{0}-j u p \cos \theta_{\mathbf{p}}\right)} \Theta\left(\cos \theta_{\mathbf{p}}\right)\right] f_{\mathrm{eq}}(x, p)
\end{aligned}
$$

and

$$
\begin{aligned}
d T_{i}^{\mu \nu}(x)= & -\frac{d x}{\lambda}\left(\frac{L}{L-x}\right) \int \frac{d^{3} p}{p^{0}} p^{\mu} p^{v} \\
& \times\left[\frac{p \cos \theta_{\mathbf{p}}}{\gamma\left(p^{0}-j u p \cos \theta_{\mathbf{p}}\right)} \Theta\left(\cos \theta_{\mathbf{p}}\right)\right] f_{\mathrm{eq}}(x, p),
\end{aligned}
$$

where the four-momentum of particles is $p^{\mu}=\left(p^{0}, \mathbf{p}\right), p=$ $|\mathbf{p}|, p^{x}=p \cos \theta_{\mathbf{p}}$, the flow velocity of the interacting matter is $u_{i}^{\mu}=\gamma(1, v, 0,0), \gamma=1 / \sqrt{1-v^{2}}, u=|v|$, and $j=$ $\operatorname{sign}(v)$.

\section{E. Changes of the conserved current and energy-momentum tensor}

In this section we show new analytical results for the changes of the conserved particle current and energymomentum tensor. The formulas are analogous to those from Refs. [10,11], but now they are calculated with the Lorentzinvariant angular factor from Eq. (7). We show results for both massive and massless particles:

$$
\begin{aligned}
d N_{i}^{0}(x)= & -\frac{d x}{\lambda}\left(\frac{L}{L-x}\right) \frac{n}{4 u^{2} \gamma^{2}}\left\{\gamma G_{1}^{-}(m)+\frac{b^{3} \Gamma(0, b)}{3 \gamma^{2}}\right. \\
& -2 \gamma b u\left[(1+j) K_{1}(a)-\mathcal{K}_{1}(a, b)\right] \\
& -u b^{2}\left[(1+j) K_{0}(a)-\mathcal{K}_{0}(a, b)\right]
\end{aligned}
$$

$$
\begin{aligned}
& \left.+\left[u^{2} \gamma^{2}(1+b)\left(u^{2}-3\right)-\frac{A(b)}{3 \gamma^{2}}\right] e^{-b}\right\} \\
& \stackrel{m=0}{\longrightarrow}-\frac{d x}{\lambda}\left(\frac{L}{L-x}\right) \frac{n}{4}\left[\frac{(3-v)(1+v)^{3}}{3} \gamma^{2}\right],
\end{aligned}
$$

$$
\begin{aligned}
d N_{i}^{x}(x)= & \frac{d N_{i}^{0}(x)}{j u}-\frac{d x}{\lambda}\left(\frac{L}{L-x}\right) \frac{n}{4 u^{2} \gamma^{2}} \\
& \times\left\{-2 j \gamma b u^{2}\left[(1+j) K_{1}(a)-\mathcal{K}_{1}(a, b)\right]\right. \\
& -j b^{2} u^{2}\left[(1+j) K_{0}(a, b)-\mathcal{K}_{0}(a, b)\right] \\
& \left.-j u \gamma^{2}\left(1+u^{2}\right)(1+b) e^{-b}\right\} \\
& \stackrel{m=0}{\longrightarrow}-\frac{d x}{\lambda}\left(\frac{L}{L-x}\right) \frac{n}{4}\left[\frac{2(1+v)^{3}}{3} \gamma^{2}\right],
\end{aligned}
$$

$$
\begin{aligned}
d T_{i}^{00}(x)= & -\frac{d x}{\lambda}\left(\frac{L}{L-x}\right) \frac{n T}{4 u^{2} \gamma^{2}}\left\{\frac{b^{4} \Gamma(0, b)}{4 \gamma^{3}}-\gamma G_{2}^{-}(m)\right. \\
& -u \gamma b^{2}\left(u^{2}+3\right)\left[(1+j) K_{2}(a)-\mathcal{K}_{2}(a, b)\right] \\
& -u b^{3}\left[(1+j) K_{1}(a)-\mathcal{K}_{1}(a, b)\right] \\
& +\gamma^{3}\left[-A(b)\left(3 u^{2}+1\right)+\frac{B(b)}{2 \gamma^{6}}-\frac{b^{2}(3+b)}{3 \gamma^{6}}\right. \\
& \left.\left.-\frac{b^{2} u^{2}(1+b)}{\gamma^{6}}+\frac{2}{3} b^{3} u^{4}\left(u^{2}-3\right)+a^{2}\right] e^{-b}\right\} \\
\stackrel{m=0}{\longrightarrow} & -\frac{d x}{\lambda}\left(\frac{L}{L-x}\right) \frac{n T}{4} \\
& \times\left[\frac{(1+v)^{4}}{2}\left(6-4 v+v^{2}\right) \gamma^{3}\right],
\end{aligned}
$$$$
d T_{i}^{0 x}(x)=\frac{d T_{i}^{00}(x)}{j u}-\frac{d x}{\lambda}\left(\frac{L}{L-x}\right) \frac{n T}{4 u^{2} \gamma^{2}}
$$$$
\times\left\{-j \gamma b^{2}\left(3 u^{2}+1\right)\left[(1+j) K_{2}(a)-\mathcal{K}_{2}(a, b)\right]\right.
$$$$
-j b\left(b^{2} u^{2}-2\right)\left[(1+j) K_{1}(a)-\mathcal{K}_{1}(a, b)\right]
$$$$
+j a b\left[(1+j) K_{0}(a)-\mathcal{K}_{0}(a, b)\right]
$$$$
-j u \gamma\left[\gamma^{2} A(b)\left(u^{2}+3\right)+(1+b)\left(u^{2}-3\right)\right.
$$$$
\left.\left.+\frac{4 b^{3}}{3} u^{2} \gamma^{2}-b^{2}\right] e^{-b}\right\}
$$$$
\stackrel{m=0}{\longrightarrow}-\frac{d x}{\lambda}\left(\frac{L}{L-x}\right) \frac{n T}{4}\left[\frac{(1+v)^{4}}{2}(4-v) \gamma^{3}\right] \text {, }
$$

$$
\begin{aligned}
d T_{i}^{x x}(x)= & \frac{d T^{0 x}(x)}{j u}-2 \frac{T}{\gamma j u}\left[d N_{i}^{x}-\frac{d N_{i}^{0}(x)}{j u}\right] \\
& -\frac{d x}{\lambda}\left(\frac{L}{L-x}\right) \frac{n T}{4 u^{2} \gamma^{2}}\left\{-\gamma u b^{2}\left(3+u^{2}\right)\right. \\
& \times\left[(1+j) K_{2}(a)-\mathcal{K}_{2}(a, b)\right]+\gamma^{3} \\
& \left.\times\left[A(b)\left(3 u^{2}+1\right)+\frac{2}{3} b^{3} u^{4}\left(u^{2}-3\right)+a^{2}\right] e^{-b}\right\} \\
\stackrel{m=0}{\longrightarrow} & -\frac{d x}{\lambda}\left(\frac{L}{L-x}\right) \frac{n T}{4}\left[\frac{3(1+v)^{4}}{2} \gamma^{3}\right],
\end{aligned}
$$




$$
\begin{aligned}
d T_{i}^{y y}(x)= & -\frac{d T^{x x}(x)}{2}-\frac{d x}{\lambda}\left(\frac{L}{L-x}\right) \frac{n T}{8 u^{2} \gamma^{2}} \\
& \times\left\{-\gamma u b^{2}\left(3+u^{2}\right)\left[(1+j) K_{2}(a)-\mathcal{K}_{2}(a, b)\right]\right. \\
& +\gamma^{3}\left[A(b)\left(3 u^{2}+1\right)-\frac{3 B(b)}{2 \gamma^{6}}\right. \\
& \left.+\frac{\left(b^{2}+1\right)(1+b)}{2 \gamma^{6}}+\frac{2}{3} b^{3} u^{4}\left(u^{2}-3\right)+a^{2}\right] e^{-b} \\
& -\frac{b^{4} \Gamma(0, b)}{\left.4 \gamma^{3}+\gamma H_{2}^{-}(m)\right\}} \\
\stackrel{m=0}{\longrightarrow} & -\frac{d x}{\lambda}\left(\frac{L}{L-x}\right) \frac{n T}{8}\left[\frac{\gamma(1+v)^{3}}{4 v^{2}}(-4+12 v\right. \\
& \left.\left.-9 v^{2}+3 v^{3}\right)\right],
\end{aligned}
$$

and

$$
d T_{i}^{z z}(x)=d T_{i}^{y y}(x)
$$

where $a=m / T, b=a \gamma, A(b)=\left(2+2 b+b^{2}\right) e^{-b}, B(b)=$ $\frac{1}{6}\left(6+6 b+3 b^{2}+b^{3}\right) e^{-b}, \quad$ and $\quad n=4 \pi T^{3} a^{2} K_{2}(a) g e^{\mu / T} /$ $(2 \pi \hbar)^{3}$ is the particle density, $g$ is the degeneracy factor; $G^{-}(m), H^{-}(m), \mathcal{K}(a, b), K(a)$, and $\Gamma(0, b)$ are defined in the Appendix. Note that the $x$-dependent factor $L /(L-x)$ is just a multiplier in these calculations, and tends to unity if we are dealing with an infinitely long FO, as in [10-12].

\section{RESULTS AND DISCUSSION}

In this section we calculate the post FO distributions and compare the results to former calculations presented in [1012]. The effect of two main differences attributed to the new Lorentz-invariant escape rate, Eq. (7), is to be checked:

(i) the infinite $(\infty) \mathrm{FO}$ layer or finite $(L) \mathrm{FO}$ layer,

(ii) the simple angular escape rate $P$ or the covariant escape rate $W$.

We performed calculations for a baryon-free massless gas, where we have used a simple EoS, $e=\sigma_{\mathrm{SB}} T^{4}$, where $\sigma_{\mathrm{SB}}=$ $\pi^{2} / 10$. The change of temperature is calculated based on this EoS and Eq. (21). There are no conserved charges in our system; consequently we use Landau's definition of the flow velocity [11]:

$$
d u_{i, \text { Landau }}^{\mu}(x)=\frac{\Delta_{i}^{\mu \nu}(x) d T_{i, v \sigma}(x) u_{i}^{\sigma}(x)}{e_{i}(x)+P_{i}(x)},
$$

where $\Delta_{i}^{\mu v}(x)=g^{\mu v}-u_{i}^{\mu}(x) u_{i}^{v}(x)$ is a projector to the plane orthogonal to $u_{i}^{\mu}(x)$, and $e_{i}(x)$ and $P_{i}(x)$ are the local energy density and pressure of the interacting component, respectively, [i.e., $T_{i}^{\mu v}(x)=\left(e_{i}(x)+\right.$ $\left.\left.P_{i}(x)\right) u_{i}^{\mu}(x) u_{i}^{\nu}(x)-P_{i}(x) g^{\mu \nu}\right]$. A detailed treatment of Eckart's flow velocity can be found in Refs. [10,11].
For such a system we finally obtain the following set of differential equations:

$$
\begin{aligned}
d \ln T & =\frac{\gamma^{2}}{4 \sigma_{\mathrm{SB}} T^{4}}\left[d T_{i}^{00}-2 v d T_{i}^{0 x}+v^{2} d T_{i}^{x x}\right], \\
d v & =\frac{3}{4 \sigma_{\mathrm{SB}} T^{4}}\left[-v d T_{i}^{00}+\left(1+v^{2}\right) d T_{i}^{0 x}-v d T_{i}^{x x}\right] .
\end{aligned}
$$

We will present the results for four different cases:

$P_{\infty}$ : We use the simple, but relativistically not invariant, angular factor $\cos \theta_{\mathbf{p}}$ in the escape rate:

$$
P_{\infty}=\frac{\cos \theta_{\mathbf{p}}}{\lambda} \Theta\left(p \cos \theta_{\mathbf{p}}\right)
$$

The system is characterized by an infinite FO length (up to $x_{\max }=300 \lambda$ in calculations). The results are shown in Figs. 8, 10, and 12. This is the same model as in [10-12]. $P_{L}$ : Next, we use the simple angular factor

$$
P_{L}=\left(\frac{L}{L-x}\right) \frac{\cos \theta_{\mathbf{p}}}{\lambda} \Theta\left(p \cos \theta_{\mathbf{p}},\right),
$$

but in this case inside a finite FO layer of $L=10 \lambda$. The results are shown in Figs. 9, 11, and 14.

$W_{\infty}$ : Then, we are dealing with the new Lorentz-invariant angular factor in the escape rate and with an infinite $\mathrm{FO}$ length $\left(x_{\max }=300 \lambda\right)$

$$
W_{\infty}=\frac{1}{\lambda}\left(\frac{p \cos \theta_{\mathbf{p}}}{p^{\mu} u_{\mu}}\right) \Theta\left(p \cos \theta_{\mathbf{p}}\right) .
$$

The results are shown in Figs. 8, 10, 12, and 13.

$W_{L}$ : Finally, we present the primary results of this paper, using both our new improvements, that is, the covariant escape rate of Eq. (7),

$$
W_{L}=\frac{1}{\lambda}\left(\frac{L}{L-x}\right)\left(\frac{p \cos \theta_{\mathbf{p}}}{p^{\mu} u_{\mu}}\right) \Theta\left(p \cos \theta_{\mathbf{p}}\right) .
$$

The results are shown in Figs. 9, 11, 14, and 15.

In the case of FO in the infinite layer the factor $L /(L-x)$ was replaced by 1 . We presented the situation at a distance of $x_{\max }=300 \lambda$, where the amount of still interacting particles is negligible.

For the particular cases when we are dealing with an infinite FO (i.e., $P_{\infty}$ and $W_{\infty}$ ) or with finite layer FO (i.e. $P_{L}$ and $W_{L}$ ), the results are plotted together. Thus, in one figure the focus is on the consequences caused by the different angular factors.

Thin lines always denote the cases with a simple relativistically not invariant angular factor; these correspond to $P_{\infty}$ and $P_{L}$. Thick lines always correspond to cases with covariant angular factor $W_{\infty}$ and $W_{L}$.

All the figures are presented in the RFF.

\section{A. The evolution of temperature of the interacting component}

The first set of figures, Figs. 8 and 9, shows the evolution of temperature of the interacting component, in fact the gradual cooling of the interacting matter, for the different cases $P_{\infty}, P_{L}, W_{\infty}$, and $W_{L}$. 


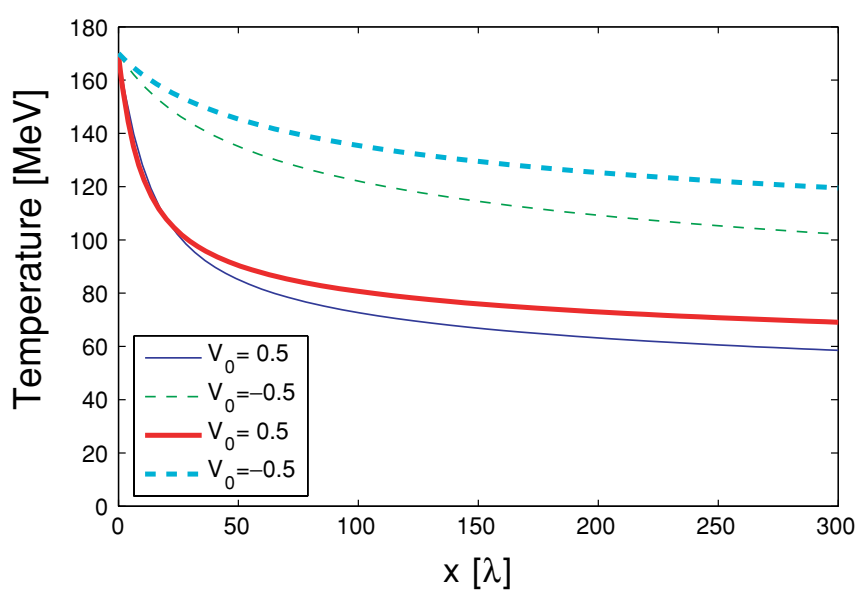

FIG. 8. (Color online) The temperature of the interacting component in the RFF for a baryon-free massless gas, calculated with the two escape rates $P_{\infty}$ (thin lines) and $W_{\infty}$ (thick lines), for an infinitely long FO $\left(x_{\max }=300 \lambda\right)$. The initial temperature is $T_{0}=170 \mathrm{MeV}$; $v_{0}$ is the initial velocity in the RFF.

First, on all figures matter with larger (positive) flow velocity $v_{0}$ cools faster. This is caused by the momentum dependence of the escape rate, which basically tells us that faster particles in the FO direction will freeze-out faster. Thus, the remaining interacting component cools down, since the most energetic particles freeze-out more often than the slow ones. Of course, for larger initial flow velocity $v_{0}$, in the FO direction, there are more particles moving in the FO direction with higher momenta on average than for a smaller flow velocity.

Now, comparing Fig. 8 with Fig. 9, we can see the difference between finite and infinite FO dynamics. In a finite layer cooling of interacting matter occurs increasingly faster as FO proceeds, whereas for FO in an infinite layer cooling gradually slows down as $x$ increases. The reason is the factor $L /(L-x)$, which speeds up FO as $L-x$ decreases and forces it to be completed within $L$.

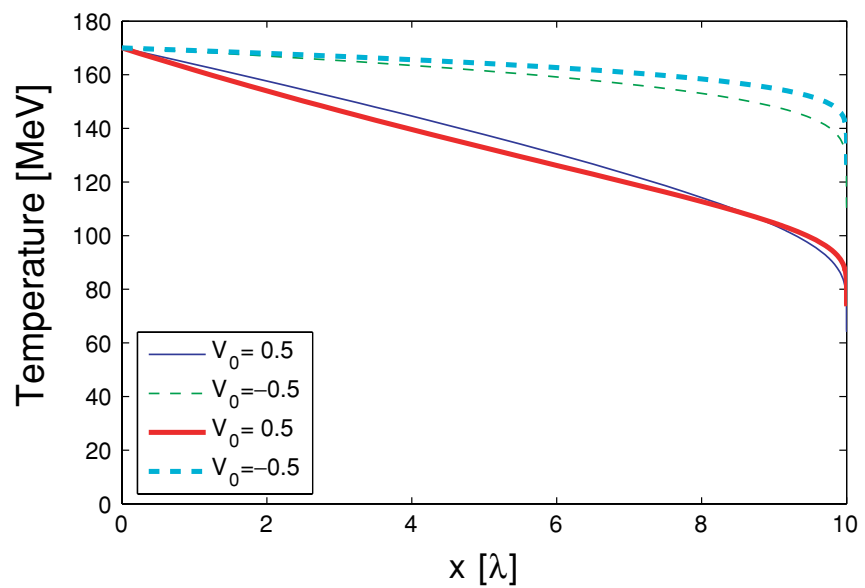

FIG. 9. (Color online) The temperature of the interacting component in the RFF, calculated with the two escape rates $P_{L}$ (thin lines) and $W_{L}$ (thick lines), for a finite $(L=10 \lambda)$ FO layer. The initial temperature is $T_{0}=170 \mathrm{MeV}$; $v_{0}$ is the initial velocity in the RFF.

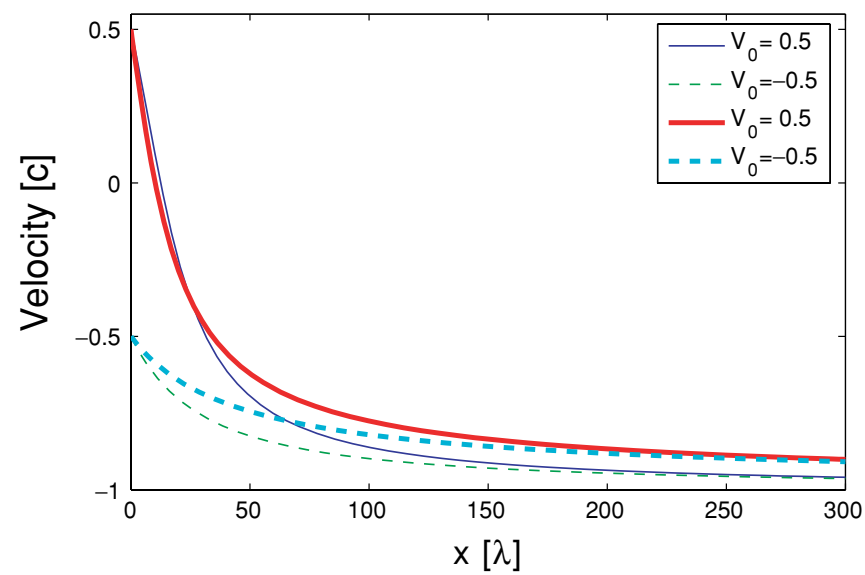

FIG. 10. (Color online) The flow velocity of the interacting component in the RFF for a baryon-free massless gas, calculated with the two escape rates $P_{\infty}$ (thin lines) and $W_{\infty}$ (thick lines), for an infinitely long FO $\left(x_{\max }=300 \lambda\right)$. The initial temperature is $T_{0}=170 \mathrm{MeV} ; v_{0}$ is the initial velocity in the RFF.

The difference between $P$ and $W$ escape rates comes from the denominator, which is $p^{0}$ for $P$ and $p^{\mu} u_{\mu}$ for $W$. This difference leads to a stronger cooling for the escape rate $P$, which is bigger than $W$, if $v_{0} \neq 0$. This can be seen well at later stages of infinitely long FO (Fig. 8), particularly for the positive initial flow velocity. In all other cases the difference between old and new angular factors is insignificant, supporting our "naive" generalization of the angular factor.

\section{B. The evolution of common flow velocity of the interacting component}

The second set of figures, Figs. 10 and 11, shows the evolution of the flow velocity of the interacting component.

In both cases the flow velocity of the interacting component tends to -1 , because the FO points in the positive direction and

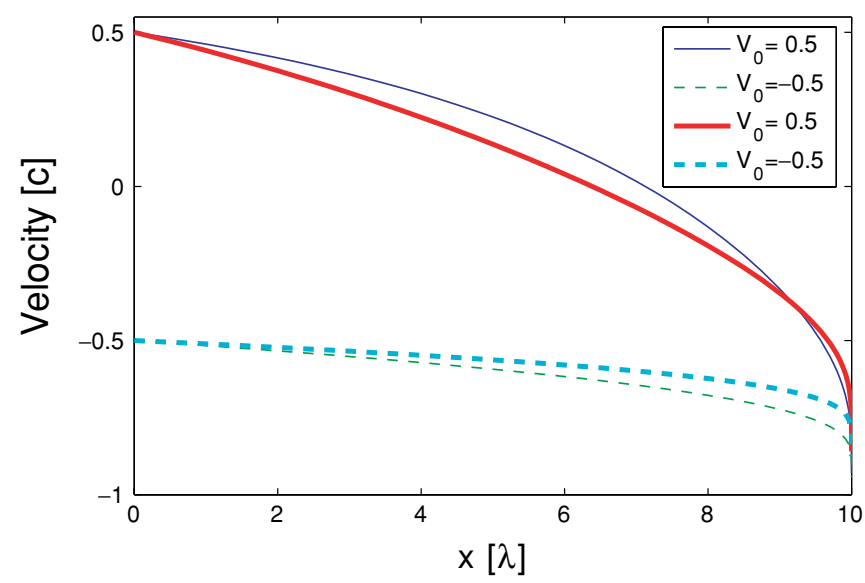

FIG. 11. (Color online) The flow velocity of the interacting component in the RFF, calculated with the two escape rates $P_{L}$ (thin lines) and $W_{L}$ (thick lines), for a finite $(L=10 \lambda)$ FO layer. The initial temperature is $T_{0}=170 \mathrm{MeV} ; v_{0}$ is the initial velocity in the RFF. 


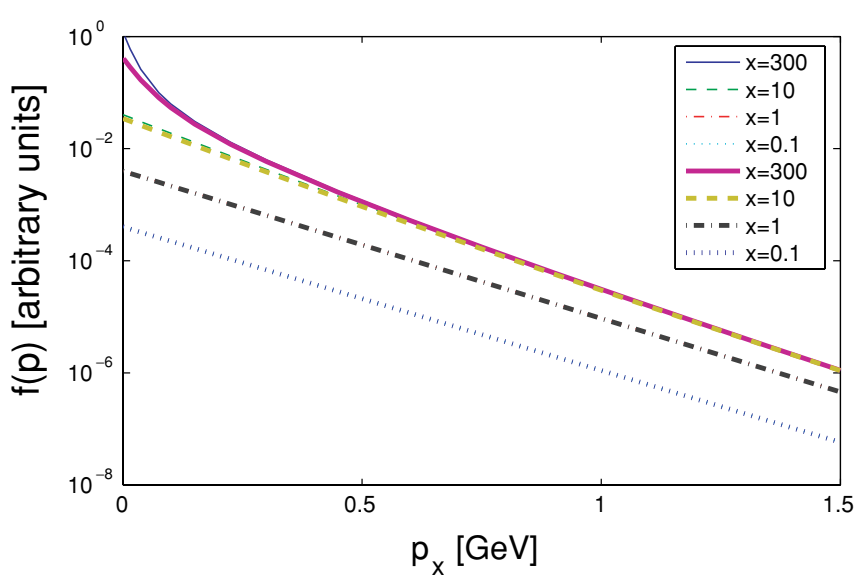

FIG. 12. (Color online) The local transverse momentum (here $p_{x}$ ) distribution for a baryon-free massless gas at $\left(p_{y}=0\right)$, calculated with the two escape rates $P_{\infty}$ (thin lines) and $W_{\infty}$ (thick lines), for an infinitely long FO $\left(x_{\max }=300 \lambda\right)$. The initial parameters are $v_{0}=0$ and $T_{0}=170 \mathrm{MeV}$. The transverse momentum spectrum is obviously curved owing to the freeze-out process. The slope of the transverse momentum distribution increases as we approach infinity.

particles with positive momenta freeze-out. Thus, the mean momentum of the rest must become negative.

Comparing Fig. 10 with Fig. 11, we can see again that in a finite layer the flow velocity decreases faster and faster as FO proceeds, whereas for FO in an infinite layer the velocity change gradually slows down as $x$ increases. The reason is the $L /(L-x)$ factor, as previously discussed.

The difference between the evolution of the flow velocity, owing to the different angular factors, is again not significant, supporting its generalization.

\section{The evolution of the transverse momentum and contour plots of the post FO distribution}

The next set of figures, Figs. 12 and 13, shows the evolution of the transverse momentum distribution, Figs. 14 and 15 present the contour plots of the post FO momentum distribution for $W_{\infty}$ and $W_{L}$. We have presented a onedimensional model here, but we assume that it is applicable for the direction transverse to the beam in heavy-ion experiments. The presented plots should be qualitatively compared to the transverse momentum distributions of measured pions.

What we see is that all the final post FO momentum distributions are essentially the same. This a is very important outcome from our analysis, which we will discuss in the following. Also, one can see that the resulting post FO distributions are nonthermal distributions, as has been shown already in [10-12]; they strongly deviate from exponential form in the low-momentum region. The increase in the final FO spectra over the thermal distribution for low momenta is connected to the fact that at late stages of the FO process, the interacting component is cold and its flow velocity is negative. So, it contributes only to the low-momentum region of the post FO spectra.

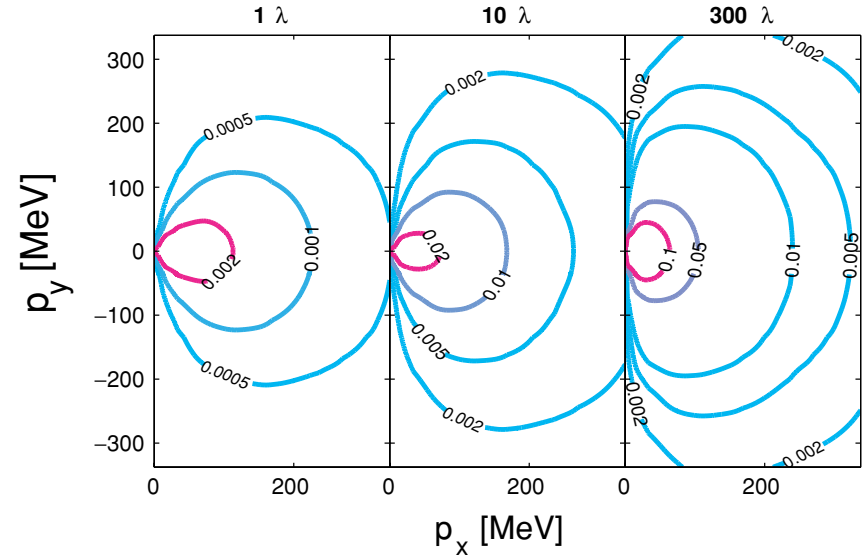

FIG. 13. (Color online) The post FO distribution, $f_{f}(x, \mathbf{p})$, in the RFF. The calculations were done with the Lorentz-invariant escape rate $W_{\infty}$ for an infinitely long FO $\left(x_{\max }=300 \lambda\right)$. The subplots correspond to $x=1 \lambda, 10 \lambda$, and $300 \lambda$, respectively. The initial parameters are $v_{0}=0$ and $T_{0}=170 \mathrm{MeV}$. Contour lines are given at values represented on the figure. The maximum is increasing with $x$ as indicated in Fig. 12. The distribution is asymmetric and elongated in the FO direction. This may lead to a large- $p_{t}$ enhancement, compared to the usual Jüttner assumption used in many earlier calculations as a post FO distribution. Note that $f_{f}(x, \mathbf{p})$ does not tend to the cut-Jüttner distribution even at very large $x$.

These results were obtained in a stationary one-dimensional model with a single flow velocity. In reality different spacetime sections of the overall FO layer are moving with respect to each other with considerable velocities (i.e., $v \approx 0.2-0.7$ ). Therefore, the superposition of these parts of the FO layer wash out the very sharp peaks at small momenta, while the curvature at higher momenta, although it is smaller, may persist even after superposition. There are several effects mentioned in the literature that can cause such a curvature. The effects discussed in this section, arising from the kinetic description, may contribute to the curvature of the spectra, but we need

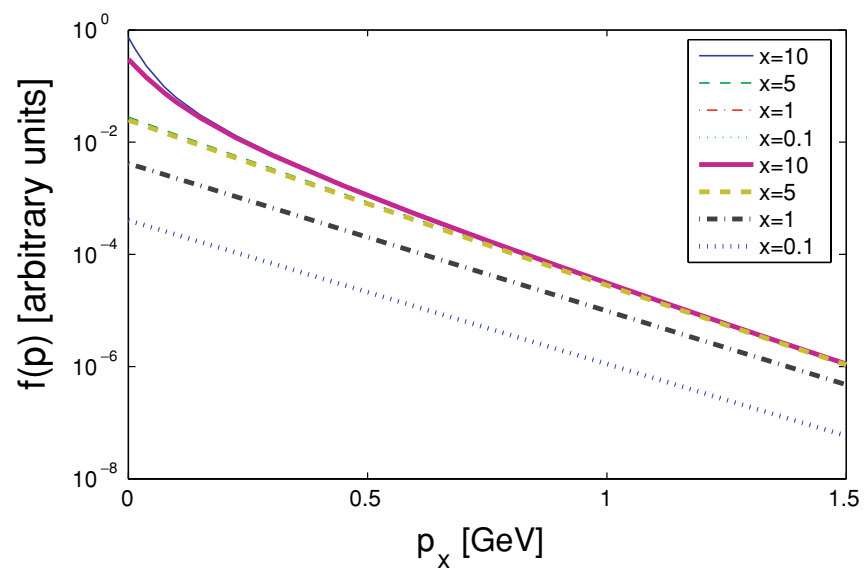

FIG. 14. (Color online) The local transverse momentum (here $p_{x}$ ) distribution for a baryon-free massless gas at $p_{y}=0$, calculated with the two escape rates $P_{L}$ (thin lines) and $W_{L}$ (thick lines) for a finite $(L=10 \lambda)$ FO layer. The initial parameters are $v_{0}=0$ and $T_{0}=170 \mathrm{MeV}$. 


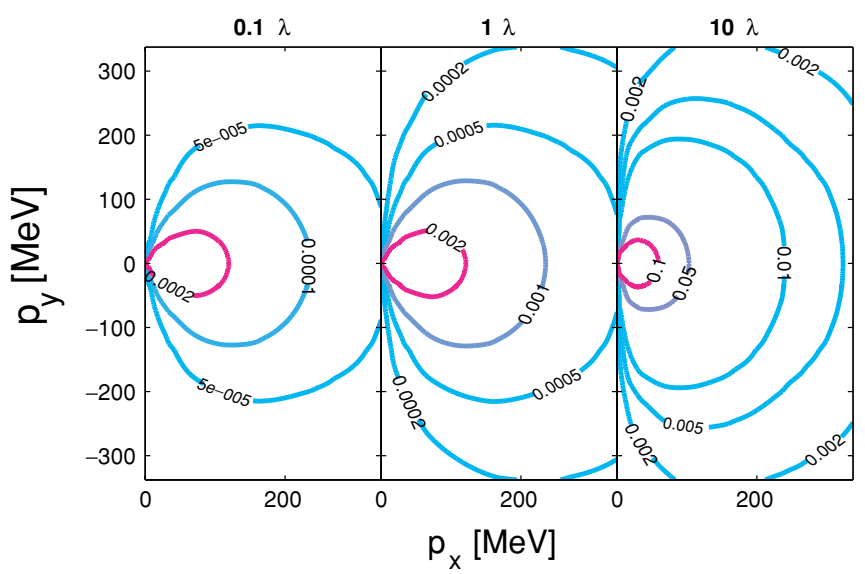

FIG. 15. (Color online) The post FO distribution, $f_{f}(x, \mathbf{p})$, in the RFF. The calculation was made using the covariant escape rate $W_{L}$ for a finite $(L=10 \lambda)$ FO layer. The subplots correspond to $x=0.1 \lambda, 1 \lambda$, and $10 \lambda$, respectively. The initial parameters are $v_{0}=0$ and $T_{0}=170 \mathrm{MeV}$. Contour lines are given at values represented on the figure.

a more realistic full scale, nonstationary three-dimensional model to estimate the expected shape of the $p_{t}$ spectra in measurements. Consequently, both the spacelike and timelike sections of the FO layer have to contribute.

\section{Freeze-out in layers of different thickness}

In this section we show the results of calculations performed with the $W_{L}$ escape rate for different finite FO layer thicknesses. Some results of such an analysis have also been presented in [26].

In Figs. 16 and 17, we present the evolution of the temperature and flow velocity of the interacting component for $L=2 \lambda, 5 \lambda, 10 \lambda$, and $15 \lambda$. We plot the resulting curves as a function of $x / L$, which allows us to present them all

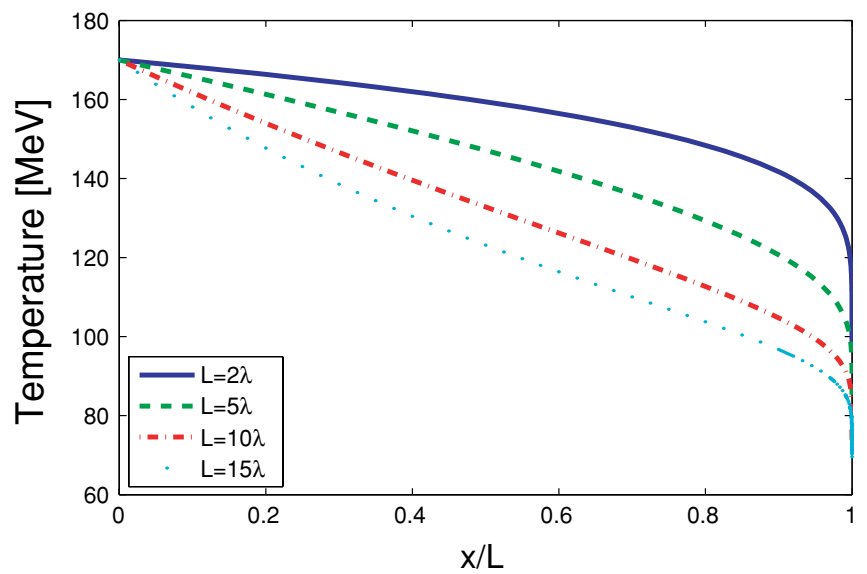

FIG. 16. (Color online) The evolution of the temperature of the interacting component in the RFF for a baryon-free massless gas, calculated with the $W_{L}$ escape rate for different FO layer thicknesses $L=2 \lambda, 5 \lambda, 10 \lambda$, and $15 \lambda$. The initial parameters are $v_{0}=0.5$ and $T_{0}=170 \mathrm{MeV}$.

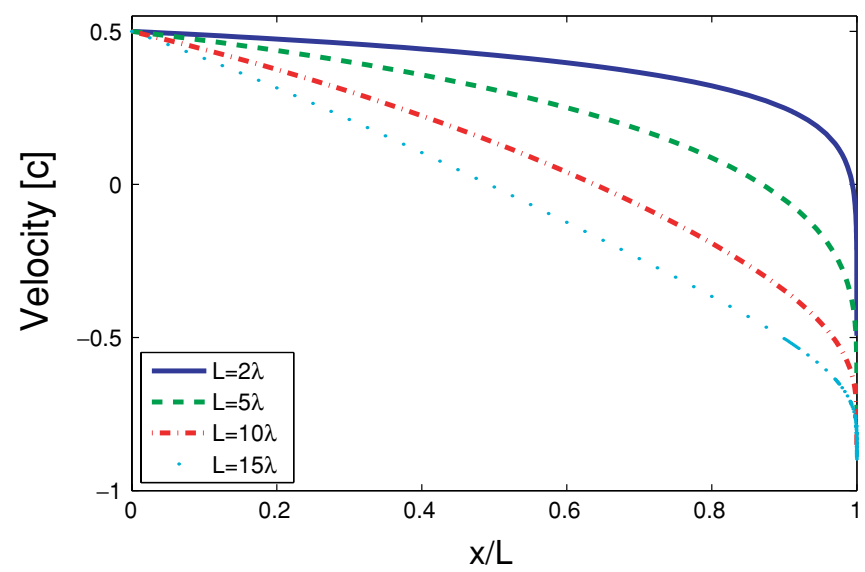

FIG. 17. (Color online) The evolution of the flow velocity of the interacting component in the RFF for a baryon-free massless gas, calculated with the $W_{L}$ escape rate for different FO layer thicknesses $L=2 \lambda, 5 \lambda, 10 \lambda$, and $15 \lambda$. The initial parameters are $v_{0}=0.5$ and $T_{0}=170 \mathrm{MeV}$.

in one figure. We clearly see, and this agrees also with our previous comparison to infinitely long FO, that by introducing and varying the thickness of the FO layer, we are strongly affecting the evolution of the interacting component.

We can also study how fast the energy density of the interacting component is decreasing (see Fig. 18). Since there is no expansion in our simple model, the evolution of the energy density is equivalent to the evolution of the total energy of the remaining interacting matter. We can see that the decrease of the energy density of the interacting component is exponentially fast, which justifies our way of getting an approximate solution for the interacting component (See Sec. II C).

Figure 19 shows the final post FO transverse momentum distribution for different $L$. Despite the differences in the evolution of the interacting component, all the final post FO distributions look the same and are practically indistinguishable.

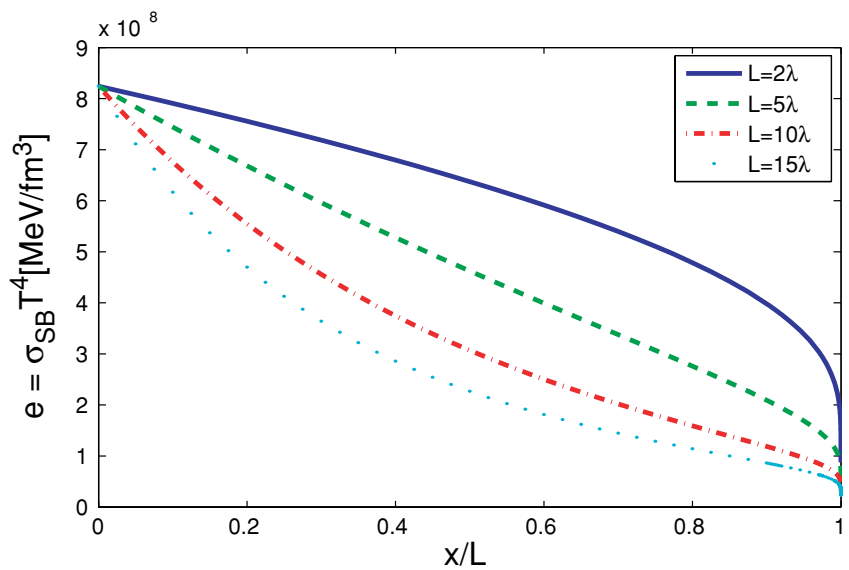

FIG. 18. (Color online) The evolution of the energy density of the interacting component in the RFF for a baryon-free massless gas, $\sigma_{\mathrm{SB}}=\pi^{2} / 10$, calculated with the $W_{L}$ escape rate for different FO layer thicknesses $L=2 \lambda, 5 \lambda, 10 \lambda$, and $15 \lambda$. The initial parameters are $v_{0}=0.5$ and $T_{0}=170 \mathrm{MeV}$. 


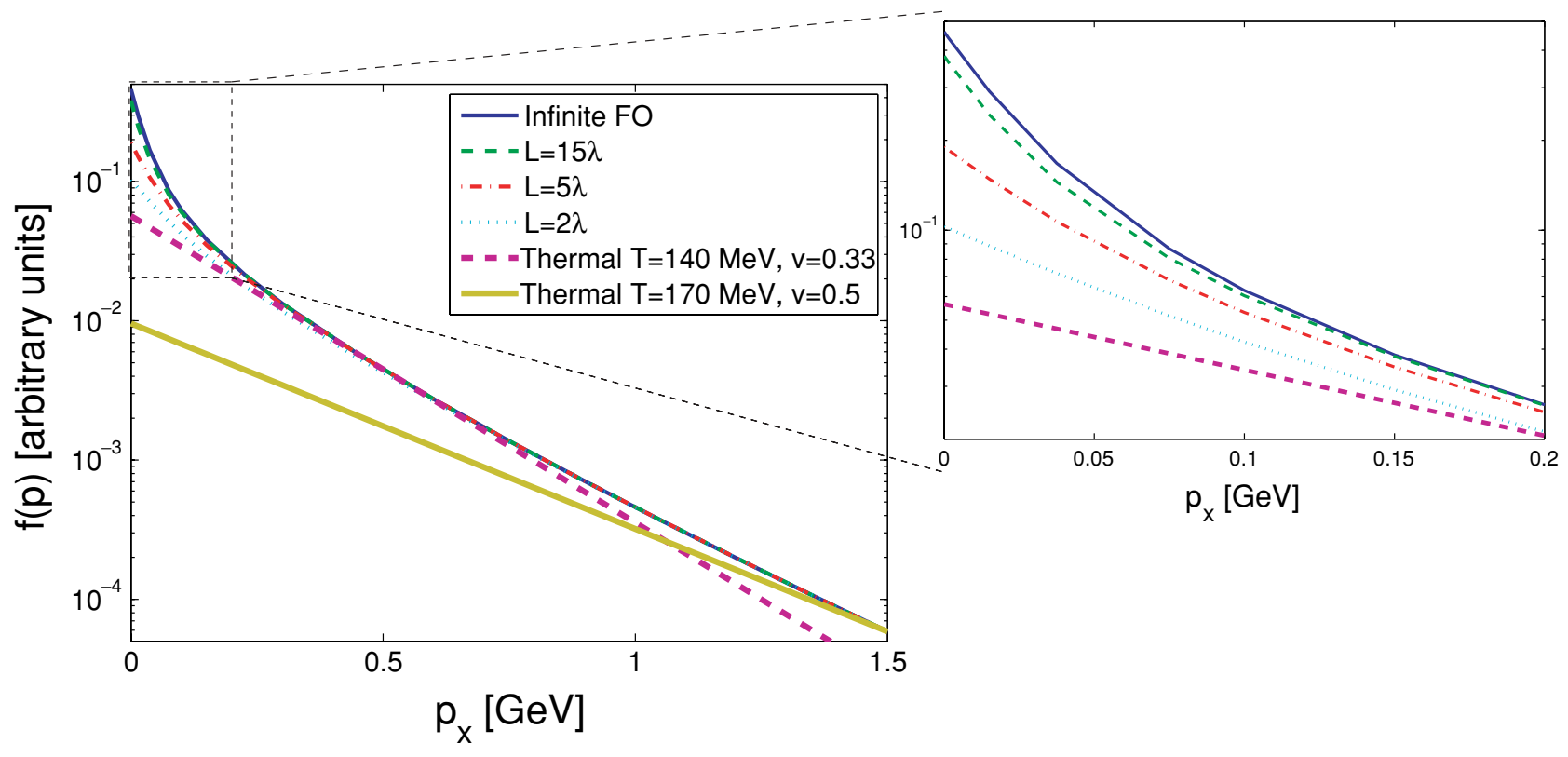

FIG. 19. (Color online) Final post FO transverse momentum (here $p_{x}$ ) distributions for the FO layers of different thicknesses. Calculations were done for baryon-free massless gas with escape rate $W_{L}$ for $L=2 \lambda, 5 \lambda$, and $15 \lambda$ and with $W_{\infty}$ (thick line). The initial conditions are the same as in Figs. 16 and 17: $T_{0}=170 \mathrm{MeV}, v_{0}=0.5$. Distributions for the different FO layer thicknesses are very similar, with some difference in the low-momenta region, which is shown in more detail in the zoomed subplot. The two thick lines correspond to some effective thermal distributions, with the corresponding parameters displayed in the plot legend. These are shown to illustrate the difference between obtained post FO distributions and thermal distributions.

The difference between the result for a FO layer as thin as $L=2 \lambda$ and that for the $L \rightarrow \infty$ limit shows up only in the low-momentum region, and it is not significant enough to allow us to resolve layers of different thicknesses from experimental spectra. Thus, the thickness of the FO layer does not affect, as we have seen already in the previous section, the final post FO distribution, which is in fact the measured quantity!

\section{CONCLUSIONS AND OUTLOOK}

In this paper we presented a simplified, but still nontrivial, Lorentz-invariant freeze-out model, that allows us to obtain analytical results in the case of a massless baryon-free gas. In addition, the model realizes FO within a finite FO layer.

We did not aim to apply directly the results presented here to the experimental heavy-ion collision data; instead our purpose was to study qualitatively the basic features of the FO effect and to demonstrate the applicability of this covariant formulation for FO in finite length.

In Figs. 8, 10, and 12 and Figs. 9, 11, and 14, we compare results with the simple, $\cos \theta_{\mathbf{p}}$, angular factor and with the Lorentz-invariant angular factor, $p^{\mu} d \sigma_{\mu} / p^{\mu} u_{\mu}$. The differences are insignificant, supporting our generalization.

As has been indicated in the previous publications [10-12], the final post FO distributions are nonequilibrated distributions, which deviate from thermal ones particularly in the low-momentum region. The final spectra have a complicated form and were calculated here numerically. In large-scale (e.g., three-dimensional CFD) simulations for spacelike FO the cancelling Jüttner distribution [27] may be a satisfactory analytical approximation.

Our analysis shows that, by introducing and varying the thickness of the FO layer, we are strongly affecting the evolution of the interacting component, but the final post FO distributions, even for small thicknesses ( e.g., $L=2 \lambda$ ), look very close to our results for an infinitely long FO, first obtained in Refs. [10-12].

The results suggest that if the measured post FO spectrum is curved, as shown in Fig. 19, then it does not matter how thick the FO layer was, and we do not need to model the details of FO dynamics in simulations of collisions! Once we have a good parametrization of the post FO spectrum (asymmetric and nonthermal), it is enough to write down the conservation laws and nondecreasing entropy condition with this distribution function [7] (and probably with some volume scaling factor to effectively account for the expansion during FO). This Cooper-Frye type of description can be viewed from two sides. From the experimental side, when we know the post FO spectra, we can extract information about the conditions in the interacting matter before FO. In theoretical (e.g., fluid dynamical) simulations such a procedure would allow us to calculate parameters of the final post FO distributions to be compared with data. In this way our results may justify the use of the FO hypersurface in hydrodynamical models for heavy-ion collisions, but with proper nonthermal post FO distributions.

At the same time, although the final distribution $f(p)$ is not sensitive to the kinetic evolution, other measurables, especially 
the two-particle correlation function, may be more sensitive to the details and extent of the FO process.

The model can also be applied to FO across a layer with a timelike normal. Several of the conclusions can be extended to the timelike case, but this requires additional studies [14].

For realistic simulations of high-energy heavy-ion reactions the full 3D description of expansion and FO of the system should be modeled simultaneously. We believe that our invariant escape rate can be a basic ingredient of such models.

\section{ACKNOWLEDGMENTS}

One of the authors, L. P. Csernai, thanks the Alexander von Humboldt Foundation for extended support in continuation of his earlier Research Award. The authors are grateful for the hospitality of the Frankfurt Institute of Advanced Studies and the Institute for Theoretical Physics of the University of Frankfurt and the Gesellschaft für Schwerionenforschung, where parts of this work were done.

E. Molnár, is grateful for the hospitality of Justus-Liebeg University of Giessen, where parts of this work were done under Contract No. HPTM-CT-2001-00223, supported by the EU-Marie Curie Training Site.

Enlightening discussions with C. Anderlik, Z. I. Lázár, and T. S. Biró are gratefully acknowledged.

\section{APPENDIX}

The definition of the $\mathcal{K}_{n}(z, w)$ function is

$$
\mathcal{K}_{n}(z, w)=\frac{2^{n} n !}{(2 n) !} z^{-n} \int_{w}^{\infty} d x e^{-x}\left(x^{2}-z^{2}\right)^{n-\frac{1}{2}},
$$

where in the case of $w=z$ and $n>-1$ this formula will lead to the modified Bessel function of the second kind, $K_{n}(z)$. Furthermore, the indefinite integral [28] is

$$
\int z^{\alpha-1} \Gamma(n, z) d z=\frac{z^{\alpha} \Gamma(n, z)-\Gamma(n+\alpha, z)}{\alpha},
$$

where $\Gamma(n, z)$ is the incomplete gamma function:

$$
\Gamma(n, z)=\int_{z}^{\infty} d t t^{n-1} e^{-t} .
$$

[1] L. D. Landau, Izv. Akad. Nauk SSSR 17, 51 (1953).

[2] A. H. Taub, Phys. Rev. 74, 328 (1948).

[3] L. P. Csernai, Sov. JETP 65, 216 (1987); Zh. Eksp. Theor. Fiz. 92, 379 (1987).

[4] K. A. Bugaev, Nucl. Phys. A606, 559 (1996).

[5] L. P. Csernai, Z. Lázár, and D. Molnár, Heavy Ion Phys. 5, 467 (1997).

[6] F. Cooper and G. Frye, Phys. Rev. D 10, 186 (1974).

[7] C. Anderlik, L. P. Csernai, F. Grassi, W. Greiner, Y. Hama, T. Kodama, Z. I. Lázár, V. K. Magas, and H. Stöcker, Phys. Rev. C 59, 3309 (1999).

[8] V. K. Magas, L. P. Csernai, E. Molnár, A. Nyíri, and K. Tamosiunas Nucl. Phys. A749, 202 (2005).
The analytically not integrable functions $G_{n}^{-}(m)$ and $H_{n}^{-}(m)$ are defined as

$$
\begin{aligned}
G_{n}^{-}(m)= & \frac{1}{T^{n+2}} \int_{0}^{\infty} d p p\left(\sqrt{p^{2}+m^{2}}\right)^{n} \\
& \times \Gamma\left(0, \frac{\gamma}{T} \sqrt{p^{2}+m^{2}}-\frac{\gamma j u p}{T}\right)
\end{aligned}
$$

and

$H_{n}^{-}(m)=\frac{1}{T^{n+2}} \int_{0}^{\infty} d p p^{n+1} \Gamma\left(0, \frac{\gamma}{T} \sqrt{p^{2}+m^{2}}-\frac{\gamma j u p}{T}\right)$.

In the massless limit, we have the $G_{n}^{-}(0)=H_{n}^{-}(0)$. Values of these functions for $(n=1,2)$ are

$$
\begin{aligned}
G_{1}^{-}(0) & =\frac{1}{T^{3}} \int_{0}^{\infty} d p p^{2} \Gamma\left(0, \frac{\gamma}{T} p(1-j u)\right) \\
& =\frac{2}{3 \gamma^{3}}(1-j u)^{-3}, \\
G_{2}^{-}(0) & =\frac{1}{T^{4}} \int_{0}^{\infty} d p p^{3} \Gamma\left(0, \frac{\gamma}{T} p(1-j u)\right) \\
& =\frac{3}{2 \gamma^{4}}(1-j u)^{-4} .
\end{aligned}
$$

In the general calculation of the integrals in RFF, we change variables from $p$ to $z$ as follows:

$$
\begin{aligned}
& \int_{0}^{\infty} d p f(p) e^{-\frac{\gamma}{T}\left(\sqrt{p^{2}+m^{2}}-j u p\right)} \\
&=j \gamma T \int_{b}^{a} d z\left(u-\frac{z}{\sqrt{z^{2}-a^{2}}}\right) e^{-z} \\
& \times f\left[\gamma T\left(j u z-j \sqrt{z^{2}-a^{2}}\right)\right] \\
&+j \gamma T \int_{a}^{\infty} d z\left(u+j \frac{z}{\sqrt{z^{2}-a^{2}}}\right) e^{-z} \\
& \times f\left[\gamma T\left(j u z+\sqrt{z^{2}-a^{2}}\right)\right]
\end{aligned}
$$

where $z=\gamma\left(\sqrt{p^{2}+m^{2}}-j u p\right) / T, a=m / T$, and $b=\gamma a$.
[9] L. P. Csernai, V. K. Magas, E. Molnár, A. Nyíri, and K. Tamosiunas, Eur. Phys. J. A 25, 65 (2005).

[10] C. Anderlik, Z. I. Lázár, V. K. Magas, L. P. Csernai, H. Stöcker, and W. Greiner, Phys. Rev. C 59, 388 (1999).

[11] V. K. Magas, C. Anderlik, L. P. Csernai, F. Grassi, W. Greiner, Y. Hama, T. Kodama, Z. I. Lázár, and H. Stöcker, Heavy Ion Phys. 9, 193 (1999).

[12] V. K. Magas, C. Anderlik, L. P. Csernai, F. Grassi, W. Greiner, Y. Hama, T. Kodama, Z. Lázár, and H. Stöcker, Phys. Lett. B459, 33 (1999); Nucl. Phys. A661, 596 (1999).

[13] V. K. Magas, A. Anderlik, C. Anderlik, and L. P. Csernai, Eur. Phys. J. C 30, 255 (2003).

[14] E. Molnár, L. P. Csernai, V. K. Magas, Z. Lázár, A. Nyíri, and K. Tamosiunas, nucl-th/0503048. 
[15] F. Grassi, Y. Hama, and T. Kodama, Phys. Lett. B355, 9 (1995).

[16] Y. M. Sinyukov, S. V. Akkelin, and Y. Hama, Phys. Rev. Lett. 89, 052301 (2002); S. V. Akkelin, M. S. Borysova, and Yu. M. Sinyukov, Acta Phys. Hung. A 22, 165 (2005).

[17] L. P. Csernai et al., in Proceedings of the NATO Advances Study Institute "Structure and Dynamics of Elementary Matter," September 22-October 2, 2003, Kemer, Turkey, hep$\mathrm{ph} / 0401005$.

[18] L. V. Bravina, I. N. Mishustin, N. S. Amelin, J. P. Bondorf, and L. P. Csernai, Phys. Lett. B354, 196 (1995).

[19] L. P. Csernai et al., J. Phys. G 31, S951 (2005).

[20] T. Csorgo and L. P. Csernai, Phys. Lett. B333, 494 (1994).

[21] L. P. Csernai and I. N. Mishustin, Phys. Rev. Lett. 74, 5005 (1995).

[22] A. Keranen, L. P. Csernai, V. Magas, and J. Manninen, Phys. Rev. C 67, 034905 (2003).
[23] J. P. Bondorf, S. I. A. Garpman, and J. Zimanyi, Nucl. Phys. A296, 320 (1978).

[24] V. K. Magas, talk at the 4th Collaboration Meeting on Atomic and Subatomic Reaction Modeling + BCPL User Meeting, Trento, Italy, February 26-29, 2004; talk at the International Workshop "Creation and Flow of Baryons in Hadronic and Nuclear Collisions," Trento, Italy, May 3-7, 2004.

[25] E. Molnar, L. P. Csernai, and V. K. Magas, in Proceedings of the 18th International Conference on Ultrarelativistic NucleusNucleus Collisions: Quark Matter 2005 (QM 2005), nuclth/0510062.

[26] V. K. Magas, L. P. Csernai, and E. Molnar, in Proceedings of the 18th International Conference on Ultrarelativistic NucleusNucleus Collisions: Quark Matter 2005 (QM 2005), nuclth/0510066.

[27] K. Tamosiunas and L. P. Csernai, Eur. Phys. J. A 20, 269 (2004).

[28] http://functions.wolfram.com/ 